Received: 26 January 2017

Accepted: 9 June 2017

Published online: 22 August 2017
NTIFIC REP

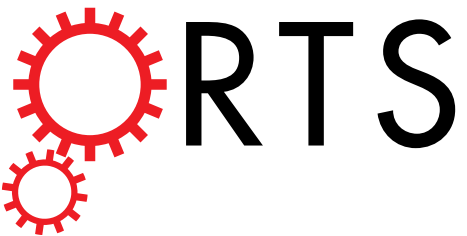

OPEN

\section{Treating the placenta to prevent adverse effects of gestational hypoxia on fetal brain development}

Tom J. Phillips ${ }^{1}$, Hannah Scott $\mathbb{D}^{1}$, David A. Menassa ${ }^{1}$, Ashleigh L. Bignell ${ }^{1}$, Aman Sood ${ }^{1}$, Jude S. Morton ${ }^{2}$, Takami Akagi ${ }^{3,4}$, Koki Azuma ${ }^{3}$, Mark F. Rogers ${ }^{5}$, Catherine E. Gilmore ${ }^{1}$, Gareth J. Inman ${ }^{6}$, Simon Grant ${ }^{7}$, Yealin Chung ${ }^{8}$, Mais M. Aljunaidy ${ }^{2}$, Christy-Lynn Cooke ${ }^{2}$, Bruno R. Steinkraus ${ }^{9}$, Andrew Pocklington ${ }^{10}{ }^{10}$, Angela Logan ${ }^{11}$, Gavin P. Collett ${ }^{12}$, Helena Kemp ${ }^{13}$, Peter A. Holmans ${ }^{10}$, Michael P. Murphy ${ }^{11}$, Tudor A. Fulga ${ }^{9}$, Andrew M. Coney ${ }^{14}$, Mitsuru Akashi $^{3,4}$, Sandra T. Davidge ${ }^{2,15}$ \& C. Patrick Case ${ }^{1}$

Some neuropsychiatric disease, including schizophrenia, may originate during prenatal development, following periods of gestational hypoxia and placental oxidative stress. Here we investigated if gestational hypoxia promotes damaging secretions from the placenta that affect fetal development and whether a mitochondria-targeted antioxidant MitoO might prevent this. Gestational hypoxia caused low birth-weight and changes in young adult offspring brain, mimicking those in human neuropsychiatric disease. Exposure of cultured neurons to fetal plasma or to secretions from the placenta or from model trophoblast barriers that had been exposed to altered oxygenation caused similar morphological changes. The secretions and plasma contained altered microRNAs whose targets were linked with changes in gene expression in the fetal brain and with human schizophrenia loci. Molecular and morphological changes in vivo and in vitro were prevented by a single dose of Mito $O$ bound to nanoparticles, which were shown to localise and prevent oxidative stress in the placenta but not in the fetus. We suggest the possibility of developing preventative treatments that target the placenta and not the fetus to reduce risk of psychiatric disease in later life.

The hypothesis for the fetal origin of adult diseases was first proposed in 1990 by David Barker ${ }^{1}$. Since then, evidence has accumulated lending credence to the hypothesis that some neuropsychiatric diseases that become symptomatic during adolescence or in adulthood have a neurodevelopmental origin ${ }^{2,3}$. Furthermore, psychological disorders such as schizophrenia, attention deficit hyperactivity disorder and autism have been associated with episodes of altered oxygen during pregnancy, as early as the end of the first trimester ${ }^{3-7}$. For schizophrenia in

${ }^{1}$ School of Clinical Sciences, University of Bristol, Southmead Hospital, Bristol, BS10 5NB, UK. ${ }^{2}$ Department of Obstetrics and Gynecology and Women and Children's Health Research Institute, University of Alberta, Edmonton, Alberta, Canada. ${ }^{3}$ Department of Applied Chemistry, Graduate School of Engineering, Osaka University, Osaka, 565-0871, Japan. ${ }^{4}$ Graduate School of Frontier Biosciences, Osaka University, Osaka, 565-0871, Japan. ${ }^{5}$ Intelligent Systems Laboratory, University of Bristol, Merchant Venturers Building, Bristol, BS8 1UB, UK. ${ }^{6}$ Division of Cancer Research, Jacqui Wood Cancer Centre, University of Dundee, Dundee, DD1 9SY, UK. ${ }^{7}$ Department of Obstetrics, Southmead Hospital, Bristol, BS10 5NB, UK. ${ }^{8}$ School of Social and Community Medicine, University of Bristol, Southmead Hospital, Bristol, BS10 5NB, UK. ${ }^{9}$ Weatherall Institute of Molecular Medicine, Radcliffe Department of Medicine, University of Oxford, Oxford, OX3 9DS, UK. ${ }^{10}$ Institute of Psychological Medicine and Clinical Neurosciences and MRC Centre for Neuropsychiatric Genetics and Genomics, Cardiff University School of Medicine, Cardiff, CF24 4HO, UK. ${ }^{11}$ MRC Mitochondrial Biology Unit, Wellcome Trust/MRC Building, Cambridge, CB2 0XY, UK. ${ }^{12}$ Nuffield Department of Obstetrics \& Gynaecology, University of Oxford, John Radcliffe Hospital, Oxford, OX3 9DU, UK. ${ }^{13}$ Department of Clinical Biochemistry, Pathology Sciences Laboratory, Southmead Hospital, Bristol, BS10 5NB, UK. ${ }^{14}$ Institute of Clinical Sciences, College of Medical and Dental Sciences, University of Birmingham, Birmingham, B15 2TT, UK. ${ }^{15}$ Department of Physiology, University of Alberta, Edmonton, Alberta, Canada. Tom J. Phillips and Hannah Scott contributed equally to this work. Correspondence and requests for materials should be addressed to C.P.C. (email: c.p.case@bristol.ac.uk) 
particular, a two-hit hypothesis has been proposed where the first 'hit' occurs in utero, disrupting neuroanatomical wiring and thereby increasing susceptibility to a second hit in later life, which triggers the disease symptoms ${ }^{8}$.

The neuropathology of neuropsychiatric disorders has been studied extensively and pathological findings generally overlap between the various disorders. For example, dendrite dysgenesis ${ }^{9,10}$ and dendritic spine pathology $^{11-16}$ of cortical neurons has been often reported in multiple brain regions including temporal, frontal and occipital cortices from autism, schizophrenia and mental retardation patients. Changes in the catecholamine system including altered tyrosine hydroxylase activity ${ }^{17,18}$, loss of parvalbumin-positive neurons ${ }^{19,20}$, and dysfunction of NMDA receptors ${ }^{9,17,21,22}$ have been observed in post-mortem brains of patients with schizophrenia and autism spectrum disorders as well as in animal models. Finally, abnormal astrocyte number or function has also been proposed as a critical change in autism and schizophrenia brains ${ }^{23-26}$.

The mechanisms by which adverse events during gestation can alter fetal development are not well understood. The placenta, which presents a double-layered interface between maternal and fetal blood during the first trimester, may play an important role ${ }^{21,27-32}$. During very early development the fetal brain's supply of the transmitter serotonin ${ }^{33}$, and possibly others ${ }^{34}$, is derived from the placenta. Obstetric challenge in the form of maternal inflammation, can alter this signalling from the placenta to the brain and disrupt axonal outgrowth of 5 HT-positive neurons within the fetal forebrain ${ }^{35}$. Furthermore preeclampsia, a pregnancy complication associated with placental insufficiency and abnormal oxygenation of the placenta, is a risk factor for the development of neuropsychiatric disease $\mathrm{e}^{36-39}$.

We have shown previously that induction of oxidative stress in the human placenta or a simple model of it, a trophoblast cell barrier, causes it to release molecules that elicit DNA damage in human fibroblasts and human embryonic stem cells ${ }^{40-42}$. Secretions from barriers exposed to hypoxia also caused morphological, electrophysiological and receptor-related changes to embryonic neurones and astrocytes, both in culture and in the developing brain in vivo ${ }^{43}$. We noted that soluble drugs such as MitoQ, a mitochondrial antioxidant, Gap26, which blocks connexin hemichannels and gap junctions, PPADS, a purinergic receptor antagonist, and C17, an antagonist of pannexin channels, could inhibit intercellular signalling between the layers of the barrier and prevent the secretion of DNA damaging factors from the barrier ${ }^{40,41}$. These observations suggest that secreted placental factors may be candidate effectors for fetal programming of disease. They present an opportunity to treat the placenta via the mother to prevent harmful signalling to the fetus.

Few drugs are used in pregnancy because of their potential adverse effects on the fetus, should they pass through the placenta. Previously we showed that nanoparticles accumulate in the top layer of a bilayered trophoblast barrier and do not pass through $\mathrm{it}^{41,42}$. Coupling a drug that stops oxidative stress in the placenta to nanoparticles could therefore prevent passage of the drug through the placenta and to the fetus. Here we investigate the potential of treating the placenta with a nanoparticle-bound MitoQ prior to a hypoxic insult to prevent damaging signalling that could affect neurodevelopment in a rat model of gestational hypoxia in vivo.

\section{Results}

Antioxidant MitoQ was successfully coupled to nanoparticles (NPs) (Supplementary Data; Supplementary Fig. S1) and tested in vitro on bilayered barriers of BeWo cells ${ }^{41}$, a choriocarcinoma cell line. MitoQ-NPs were most effective at reducing the effects of in vitro hypoxia, compared to NP-bound Gap26 or PPADS (Supplementary Fig. S2). When NPs were applied to BeWo barriers for up to $24 \mathrm{~h}$, they were predominantly located in the top layer of the barrier (Fig. 1a) ${ }^{40}$. There was no evidence of passage of NPs, or release of MitoQ, across the barriers into the media below except at a very high NP dose $(2 \mathrm{mg} / \mathrm{ml})$ (Fig. 1c; Supplementary Table S1). For subsequent in vivo experiments a 2580-fold lower dose was selected, at which NPs were not observed to cross the in vitro barrier.

We investigated if treatment with MitoQ-NPs would be able to prevent placental oxidative stress, altered signalling from the placenta and neuroanatomical changes in the offspring in an established in vivo model of gestational hypoxia ${ }^{44}$. Briefly, pregnant rats were exposed to $11 \%$ oxygen for the last 6 days of pregnancy. This exposure reduces birth-weight and is known to cause abnormal fetal cardiovascular programming ${ }^{30,45}$. We used a single dose of MitoQ-NPs, injected intravenously into the dam at the start of the hypoxic exposure, as the least invasive procedure.

Effects of MitoQ-NPs on placenta and fetus. As expected ${ }^{30,45}$, birth-weights were decreased following maternal hypoxia (Fig. 2a). Maternal MitoQ-NP injection rescued over $60 \%$ of this deficit. Hypoxia and MitoQ-NPs had no effect on body weight at P30, placental weight or brain weight (Fig. 2a; Supplementary Fig. S3).

We investigated if NPs could reach the placenta in vivo and whether maternal hypoxia or MitoQ-NP injection caused changes to the placenta. NPs were detected within the placenta, most prevalently in the labyrinth but also in the junctional zone (Fig. 2b). The NPs were particularly found in cytotrophoblasts, which face the maternal circulation, and less commonly seen in syncytiotrophoblast cells, which face the fetus. NPs were not found in the fetal brain or in thoracic or abdominal tissues including liver (Fig. 2b). NPs were detected in the maternal liver, particularly in Kupffer cells and hepatocytes (Supplementary Fig. S3). Areas of NP localisation were observed sparsely throughout the maternal brain. Within these areas, fluorescence was mainly detected within neuronal processes (Supplementary Fig. S3).

Neither maternal hypoxia nor NP injection caused a change in placental fine structure (Fig. 2c) or in the width of labyrinth or decidua (Supplementary Fig. S3). Nor was the fetal capillary network altered in the labyrinth (Fig. 2d; Supplementary Fig. S3). Levels of oxidative stress were significantly increased in the labyrinth and junctional zones of the placenta following gestational hypoxia. Treatment with MitoQ-NPs significantly reduced levels of oxidative stress (Fig. 2e; Supplementary Fig. S3). Analysis of maternal liver and fetal liver showed elevated levels of oxidative stress following maternal hypoxia. After MitoQ treatment increased oxidative stress was still 
a

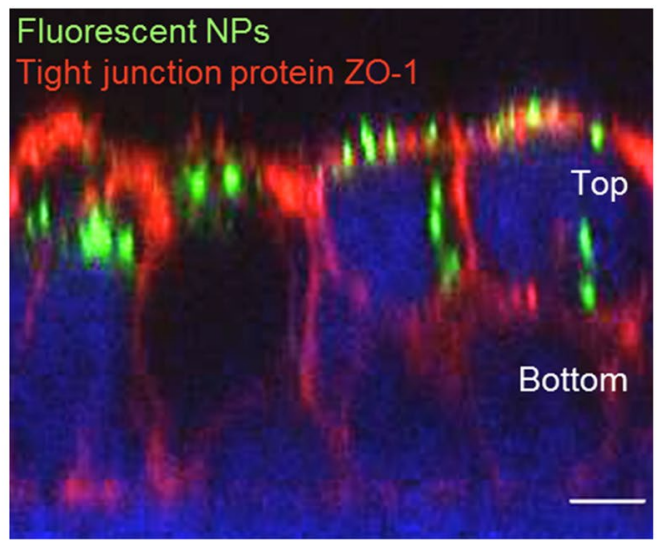

b

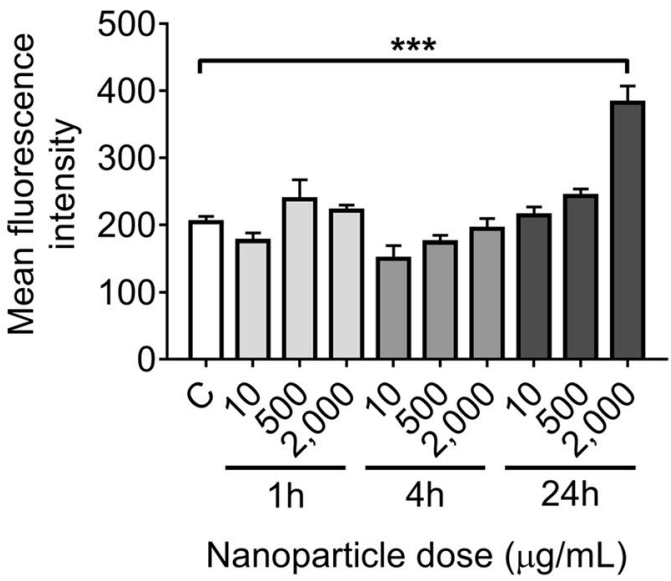

Figure 1. Characterisation of MitoQ-NPs. (a) Confocal images of bilayered BeWo barriers $24 \mathrm{~h}$ after application of $2 \mathrm{mg} / \mathrm{mL}$ fluorescent NPs (green) to the top of the barrier. Tight junction protein ZO-1 is labelled in red, nuclei in blue. Scale bar $=10 \mu \mathrm{m}$. (b) Levels of fluorescence detected in the tissue culture medium below the bilayered BeWo barriers up to $24 \mathrm{~h}$ after application of different doses of NPs to the top of the barrier $(n=2)$. $* * * p<0.001$ as determined by post-hoc testing following one-way ANOVA with Bonferroni correction for multiple comparisons.

observed in fetal liver but not in maternal liver (Fig. 2e). No changes in oxidative stress were detected in the fetal brain after maternal hypoxia or NP treatment (Fig. 2e; Supplementary Fig. S3).

These observations suggest that the NPs injected into the maternal circulation reduce oxidative stress in maternal tissues and placenta but not in fetal tissues following gestational hypoxia. Moreover, they rescue fetal birth-weight although they do not reach the fetus.

Effects of MitoQ-NPs on placental secretions. Several authors have stressed the importance of the placenta, as the interface between mother and fetus, in fetal programming ${ }^{21,27-32}$. We hypothesised that secretions from the placental barrier in response to hypoxia, as observed previously in vitro ${ }^{43}$, might play a role in fetal development in vivo. By targeting oxidative stress in the placenta, MitoQ-NPs may prevent potentially damaging signalling from the placenta. We tested the hypothesis that MitoQ-NPs could alter the secretion of molecules from the placenta by investigating microRNAs (miRNAs), proteins including bone morphogenetic proteins (BMPs) and amino acids, all of which have potential relevance to fetal development ${ }^{46-49}$, psychiatric diseases ${ }^{50-52}$ and placental hypoxia ${ }^{53,54}$. Levels of these molecules were measured in fetal plasma and in culture medium conditioned ex vivo by placental tissue ('rat placenta conditioned medium'), collected from rats exposed to gestational hypoxia and MitoQ-NP treatment. Additionally, we analysed culture medium conditioned in vitro by bilayered barriers of BeWo cells ('BeWo barrier conditioned medium') following exposure to altered oxygen and MitoQ-NPs.

Changes to oxygen levels altered total levels of miRNAs, small RNAs and potentially small extracellular vesicles, such as exosomes, released from BeWo barriers (Fig. 3a; Supplementary Fig. S4). There was no change in total miRNA or small RNA in medium conditioned by rat placenta after gestational hypoxia (Fig. 3b; Supplementary Fig. S4). However in media conditioned by BeWo barriers or rat placenta and in fetal plasma there was a complex pattern of increased and decreased levels of individual miRNA (Fig. 3c-e; Supplementary Fig. S4). The application of MitoQ-NPs partially prevented these changes for the majority of differentially secreted miRNAs.

The pattern of secretion of BMPs from BeWo barriers was also altered following exposure to reduced oxygen (Fig. 3f). This was prevented by the MitoQ-NPs. The proteome profile of fetal plasma was changed in response to gestational hypoxia (Fig. 3h,j). Proteins with altered abundance were significantly enriched for exosomes and lipoproteins (Fig. 3k,1). No change in protein abundance was seen in the fetal plasma after maternal MitoQ-NP treatment (Fig. $3 \mathrm{~h}, \mathrm{j})$. In contrast, in rat placenta conditioned media, there was little change in protein levels after gestational hypoxia but significant change after MitoQ-NP treatment (Fig. 3g,i).

Levels of amino acids in rat placenta conditioned media were not significantly altered in response to maternal hypoxia (Supplementary Tables S2-4; Supplementary Fig. S4).

Taken together these results indicate that gestational hypoxia selectively alters the secretion of miRNAs and proteins in vivo, ex vivo and in vitro. Maternal administration of MitoQ-NPs 'normalises' the miRNA secretion patterns in a global manner.

Effects of MitoQ-NPs on gene expression in the offspring brain. Having identified factors, such as miRNAs, which were differentially secreted from the placental barrier following a hypoxic insult, we wanted to investigate if these had the potential to play a role in shaping the brain during fetal development. miRNAs regulate mRNA abundance ${ }^{55}$ and have been shown to be involved in neurodevelopment and disease ${ }^{56}$. Moreover exosomes, extracellular vesicles known to carry miRNAs, can cross the blood-brain barrier and reach the brain ${ }^{57}$. 
a
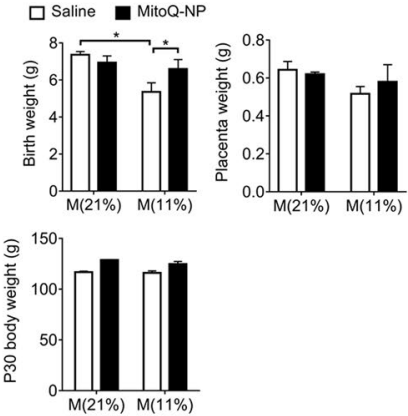

C

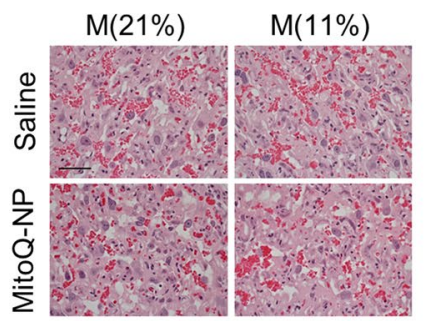

b

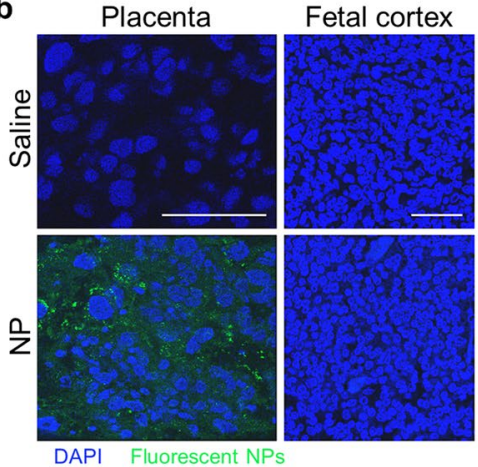

Fetal liver

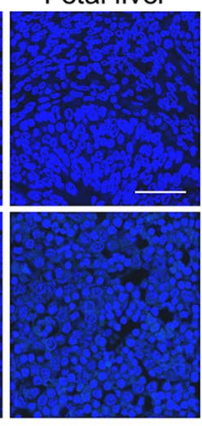

d

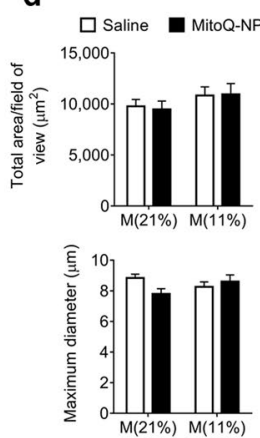

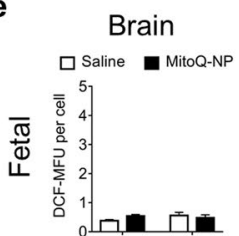

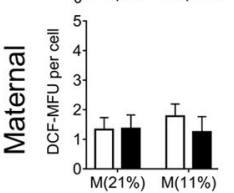

Liver

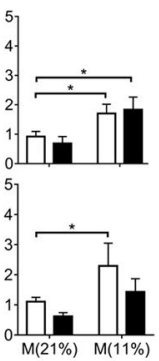

Placenta

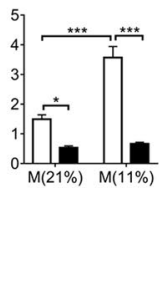

Figure 2. Effects of MitoQ-NPs on the rat in vivo. (a) Birth-weight (top left; $n=6$ litters), placenta weight (top right; $n=6$ litters) and body weight at $\mathrm{P} 30$ (bottom; $n=24$ individuals) of offspring exposed to maternal normoxia, $\mathrm{M}(21 \%)$, or hypoxia, $\mathrm{M}(11 \%)$, preceded by maternal administration of saline or MitoQ-NPs. (b) Confocal images of rat placental labyrinth, fetal cortex and fetal liver at GD16 after maternal injection with saline or fluorescent NPs (green), counterstained with DAPI (blue), scale bar $=100 \mu \mathrm{m}$. (c) Light micrographs showing the gross histology (H\&E stain) of the placental labyrinth at GD20 following in vivo normoxia or hypoxia with maternal saline or MitoQ-NP injection (scale bar $=2 \mu \mathrm{m}$ ). (d) Analysis of total area per field of view (top) and maximum diameter (bottom) of placental blood vessels after gestational hypoxia combined with maternal injection of saline or MitoQ-NPs ( $n=3$ placenta per condition, each from a different dam). (e) Levels of fluorescent dichlorofluorescein (DCF) as a measure of reactive oxygen species and thus oxidative stress in fetal brain (left, top) and liver (middle, top), maternal brain (left, bottom) and liver (middle, bottom) and placenta (right) after exposure to altered oxygen in vivo, with or without maternal MitoQ-NP injection (biological replicates: fetal and placental samples, $n=6$; maternal samples, $n=3$ ). Additional significant differences for 'placenta' graph: $\mathrm{M}(21 \%)+$ saline vs $\mathrm{M}(11 \%)+$ MitoQ-NP, $p<0.05 ; \mathrm{M}(21 \%)+$ MitoQ-NP vs $\mathrm{M}(11 \%)+$ saline, $p<0.001$. ${ }^{*} p<0.05, * * * p<0.001$ as determined by post-hoc testing following ANOVA.

Using bioinformatics analyses we investigated the predicted target genes of those miRNAs that were differentially expressed in rat placenta conditioned media and in fetal plasma, in response to gestational hypoxia. The predicted target genes were enriched in the brain compared to other organs $(\mathrm{p}<0.001)$ (Fig. $4 \mathrm{a}, \mathrm{d})$ as well as in biological processes linked to development (Fig. 4b,e). Furthermore, predicted miRNA targets were enriched within copy number variants (CNVs) found in schizophrenia cases compared to CNVs found in matched controls (Fig. 4c,f; Supplementary Table S5). An additional analysis conditioned on a minimal set of CNS-linked gene-sets previously shown to be enriched for schizophrenia CNVs in the same case-control data ${ }^{58}$; this indicated that while some of the enrichment seen in our predicted targets was shared with these known associated gene sets, a significant proportion seems to be independent (Supplementary Table S6). These results suggest that the differentially secreted miRNAs could target genes involved in neurodevelopment and neurodevelopmental disease.

Next we investigated if gene expression was altered in the fetal brain exposed to gestational hypoxia and if these changes may be correlated with the changes in secreted miRNAs. The mRNA levels of 510 genes were significantly altered in the fetal brain after gestational hypoxia. Maternal MitoQ-NP administration reduced these effects (Fig. 4g). Genes down-regulated following hypoxia were enriched for developmental processes (Fig. 4h). Differentially expressed genes in the fetal brain were significantly enriched for predicted targets of those miRNAs that had been found to be significantly altered in fetal plasma and conditioned medium (Supplementary Table S7), suggesting a potential link between secreted miRNAs and cortical transcriptome changes following a hypoxic insult. For a subset of significant miRNAs, including miR-17-5p (Fig. 4i), negative correlation with one or more significant mRNA changes in the brain was detected (Table 1). 

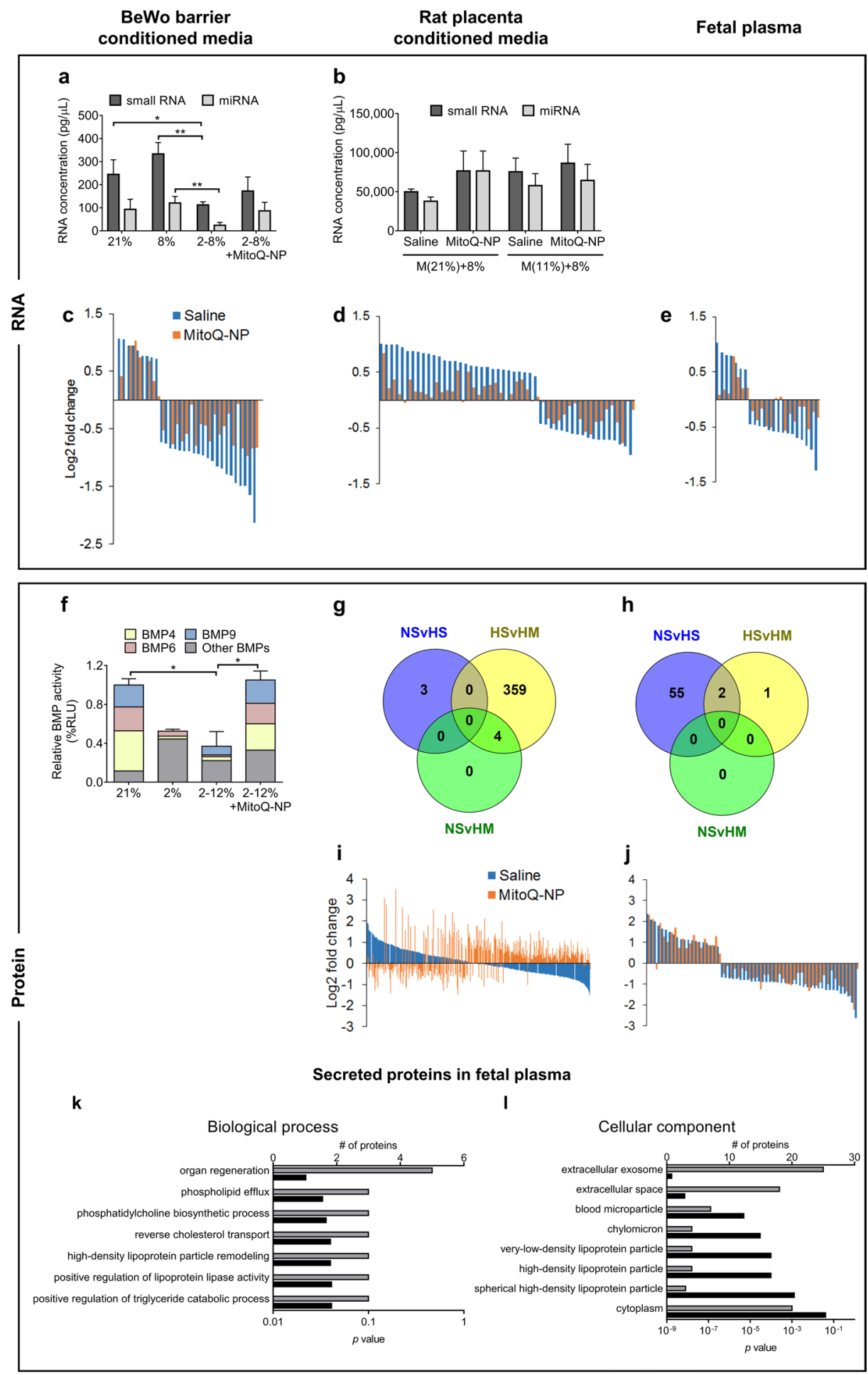

Figure 3. Effects of MitoQ-NPs on secreted molecules from BeWo barriers and placenta and into fetal blood. Levels of molecules in media conditioned by bilayered BeWo barriers $(\mathbf{a}, \mathbf{c}, \mathbf{f})$ or rat placenta $(\mathbf{b}, \mathbf{d}, \mathbf{g}, \mathbf{i})$, and in fetal plasma (e,h,j-l). BeWo barriers had been exposed to $21 \%$ oxygen, to $8 \%$ oxygen or to $2 \%$ oxygen followed by $8 \%$ oxygen to mimic hypoxia-reoxygenation (2-8\%), with or without application of MitoQ-NPs. Placentas and fetal plasma were collected from rats exposed to gestational normoxia, $\mathrm{M}(21 \%)$, or hypoxia, $\mathrm{M}(11 \%)$, after maternal administration of saline or MitoQ-NPs. Placentas were incubated ex vivo for $24 \mathrm{~h}$ at $8 \%$ oxygen before collection of conditioned medium. (a,b) Total levels of small RNAs and miRNAs. (c-e) Log2-fold changes of miRNAs with significantly $(p<0.05)$ different secretion under altered oxygen alone (blue) or altered oxygen plus MitoQ-NP application (orange), relative to control levels (normoxia + saline). (f) Relative activity of BMPs in relative luminescence unit (RLU), normalised to $21 \%$. Significant differences not shown in graph: BMP4, $21 \%$ vs $2 \%$, $p=0.01,21 \% \mathrm{vs} 2-12 \%, p=0.04$; Other BMPs, $21 \% \mathrm{vs} 2 \%, p=0.004 ; 21 \% \mathrm{vs} 2-12 \%, p=0.04$. (g,h) Levels of significant $(p<0.05)$ differentially abundant proteins in rat placenta conditioned medium $(\mathbf{g})$ and fetal plasma 
(h). Differences were analysed between: normoxia + saline and hypoxia + saline (NSvHS); hypoxia + saline and hypoxia + MitoQ-NPs (HSvHM); normoxia + saline and hypoxia + MitoQ-NPs (NSvHM). (i) Log2-fold changes of proteins detected in rat placenta-conditioned medium under altered oxygen alone (blue) or altered oxygen plus MitoQ-NP application (orange), relative to control (normoxia + saline). Proteins depicted showed significant differential abundance in the hypoxia + MitoQ-NP conditioned compared to hypoxia + saline. $n=3$ biological replicates, each from a different litter. (j) Log2-fold changes of proteins with significantly different abundance in fetal plasma under altered oxygen alone (blue) or altered oxygen plus MitoQ-NP application (orange), relative to control levels. $n=4$ for groups normoxia + saline, normoxia + MitoQ-NPs and hypoxia + MitoQ-NPs; all others, $n=3$. (k,l) Gene ontology analysis of significant differentially abundant proteins in fetal plasma after exposure to maternal hypoxia compared to normoxia. Enrichment of biological processes (k) and cellular compartments (l) is shown. Grey bars represent number of proteins assigned to each gene ontology term and black bars show respective $p$ value. ${ }^{*} p<0.05$, $* * p<0.01$, Tukey's post-hoc test following one-way or two-way ANOVA.

The results indicate that maternal MitoQ-NP treatment can partially prevent hypoxia-induced gene expression changes in the fetal brain and point towards a potential link between miRNAs secreted from the placenta and the transcriptome of the developing brain.

Neurodevelopmental effects of MitoQ-NPs in vitro and in vivo. The placenta may play an important role in the aetiology of neurodevelopmental disorders ${ }^{21,30-32}$. Therefore, we further explored the potential link between placental secretions and changes in the offspring brain. We examined whether fetal plasma and media conditioned by BeWo barriers or rat placenta had similar biological effects on embryonic cortical neurons in culture. We further investigated if these effects might also be noted in vivo within the offspring brains at a later stage of development. We chose parameters that have relevance to psychological disorders ${ }^{9,17,24,59}$.

In cortical cultures, exposure to conditioned media and plasma collected following a hypoxic episode caused a shortening of dendrites (Fig. 5a-c; Supplementary Fig. S5), a reduction of tyrosine hydroxylase (TH)-positive process lengths (Fig. 5f,g; Supplementary Fig. S5), a loss of GluN1 receptor subunit staining (Fig. 5j-1; Supplementary Fig. S5) and an increase in astrocyte-to-neuron ratio (Fig. 5n-p; Supplementary Fig. S5). In general, such changes were also seen in the brain (Fig. $5 \mathrm{~d}, \mathrm{~m}, \mathrm{q}$ ). A decrease in dendrite length was restricted to the thalamic reticular nucleus (TRN; Fig. 5d) although thick, abnormally branched dendrites were noted in the somatosensory cortex (SSC; Fig. 5r). Process lengths of $\mathrm{TH}^{+}$neurons were increased in cortex (CTX) and TRN (Fig. 5i), which contrasted with the reduction in process length observed after exposing cortical cultures to fetal plasma or rat placenta conditioned medium (Fig. $5 \mathrm{f}, \mathrm{g}$ ). However, the directionality of the effect observed in vivo was replicated when cortical cultures were exposed to BeWo conditioned medium (Fig. 5e) and when neuron-only cultures were exposed to media conditioned by rat placenta (Fig. $5 \mathrm{~h}$ ).

In general, the actions of MitoQ-NP treatment were also similar across the different models and either significantly prevented the effects of hypoxia, or caused hypoxia to no longer have significant effects, on dendrite length (Fig. 5a-d; Supplementary Fig. S5), $\mathrm{TH}^{+}$cell process length (Fig. 5e-i), GluN1 receptor subunit staining (Fig. 5j-m; Supplementary Fig. S5) and astrocyte-to-neuron ratio (Fig. 5n-q). Additionally, the numerical density of parvalbumin-containing cells was significantly reduced in TRN, CTX and SSC of offspring following maternal hypoxia and rescued in the TRN by MitoQ-NP injection (Fig. 5s). Total cell count was not affected by either hypoxia or MitoQ-NP application in vitro or in the TRN, whereas a reduction in cell number was observed in the CTX (Supplementary Fig. S6). The number of $\mathrm{TH}^{+}$cells were reduced in cortical cultures only after additional severe hypoxia was applied to the placenta ex vivo and no change was observed in vivo (Supplementary Fig. S6).

Recently it was demonstrated that lipopolysaccharide, which shows minimal transport across the blood-brain barrier, may nonetheless have toxic actions within the brain by acting on astrocytes located at the blood-brain barrier $^{60}$. We noted previously that glutamate may play a role in mediating the effects of hypoxia-conditioned media on cortical cultures ${ }^{43}$. We therefore tested the effects of media conditioned by rat placenta after maternal hypoxia on astrocyte signalling to neurons with and without glutamate blockade (Fig. 6). Dendrite lengths and GluN1 staining intensity were decreased in mixed astrocyte/neuron cultures (Fig. 6a,g), unchanged in neuron-only cultures (Fig. 6b,h) and decreased if astrocyte cultures were first exposed and then this astrocyte-conditioned medium was applied to neuron-only cultures (Fig. 6c,i). Moreover these effects were blocked by the NMDA receptor antagonist $\mathrm{MK}-801$. In contrast, $\mathrm{TH}^{+}$cell processes, although decreased in mixed cultures (Fig. 6d) and after exposure to the astrocyte-conditioned media (Fig. 6f), were increased in neuron-only cultures, an effect that was not altered by the glutamate antagonist (Fig. 6e).

Together these results suggest that maternal administration of MitoQ-NPs can ameliorate some of the long-term effects of gestational hypoxia on the offspring brain. These effects can be replicated in cortical cultures via exposure to rat placenta conditioned media or fetal plasma and may involve astrocyte-to-neuron signalling, partially via glutamate.

\section{Discussion}

We showed that maternal injection of a single dose of NP-bound antioxidant MitoQ, during exposure to gestational hypoxia, reduced oxidative stress in the placenta and prevented a reduction of birth-weight (Supplementary Fig. S7). Birth-weight is a strong predictor for disease in later life ${ }^{61}$ and low birth-weight has been associated with altered neuropsychiatric outcomes ${ }^{2}$. Importantly the nanoparticle-bound drug appeared to exert its effect on the fetus indirectly, without passing through the placenta to the fetus. This is supported by the lack of nanoparticles detected in the fetus, the lack of MitoQ in conditioned media from BeWo barriers or rat placenta along with the 

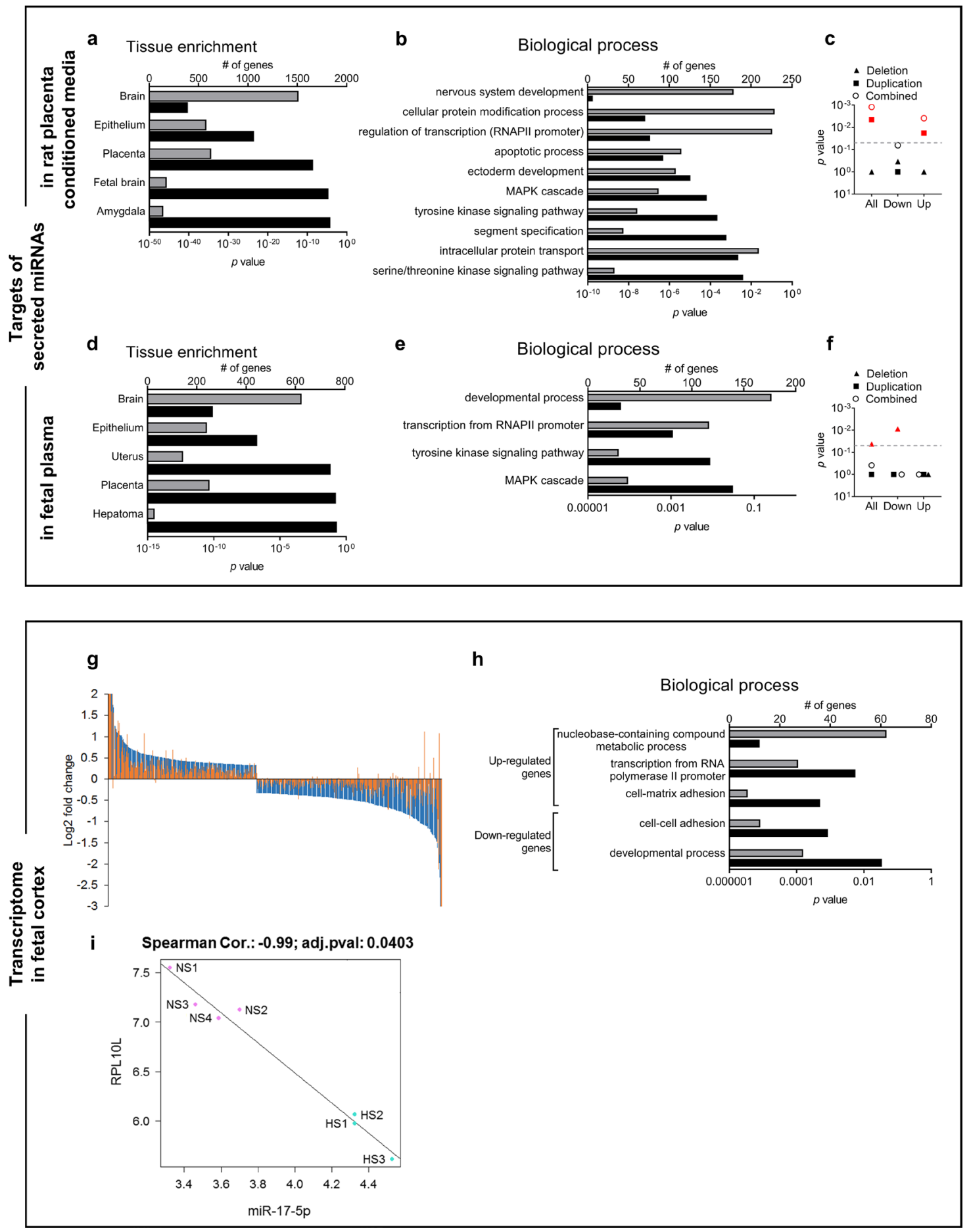

Figure 4. Characterisation of secreted miRNAs and transcriptome changes in the fetal brain. (a-f) Predicted targets of miRNAs that were significantly altered in response to maternal hypoxia in conditioned medium from rat placenta $(\mathbf{a}-\mathbf{c})$ or in fetal plasma $(\mathbf{d}-\mathbf{f})$ were analysed for enrichment of tissue types $(\mathbf{a}, \mathbf{d})$ and of biological processes $(\mathbf{b}, \mathbf{e})$. Predicted targets of all miRNAs, of upregulated miRNAs and of downregulated miRNAs were investigated for enrichment in CNVs associated with schizophrenia. Significant enrichment at $p<0.05$ (grey line) shown in red $(\mathbf{c}, \mathbf{f})$. (g-i) Changes in the transcriptome were measured in the fetal cortex of offspring exposed to gestational normoxia or hypoxia and maternal administration of saline or MitoQ-NPs. Log2-fold changes of significant differentially expressed genes under hypoxia alone (blue) or hypoxia plus MitoQ-NP application (orange), relative to control levels (normoxia + saline) are shown (g). Significantly enriched biological processes of differentially expressed genes; the grey bars represent number of proteins assigned to each gene ontology term and the black bars show the respective $p$ value (h). Correlation of miR17-5p abundance changes in fetal plasma with expression changes of the gene coding for Ribosome protein L10-like (RPL10L) in fetal brain, under Normoxia + Saline (NS) and Hypoxia + Saline (HS) conditions (i). Logtransformed counts are shown. $p$ value was adjusted for multiple comparisons using Benjamini-Hochberg. 


\begin{tabular}{|l|l|l|l|l|l|}
\hline & mRNA & $\begin{array}{l}\text { miRNA } \\
\text { log2 FC }\end{array}$ & $\begin{array}{l}\text { mRNA log2 } \\
\text { FC }\end{array}$ & $\begin{array}{l}\text { Correlation } \\
\text { coefficient }\end{array}$ & $\begin{array}{l}\text { Adjusted } \boldsymbol{p} \\
\text { value }\end{array}$ \\
\hline \multirow{4}{*}{ rno-miR-1 } & HSPA5 & -0.92 & 0.71 & -0.992 & 0.036 \\
\cline { 2 - 6 } & PDIA4 & -0.92 & 0.64 & -0.993 & 0.035 \\
\cline { 2 - 6 } & XBP1 & -0.92 & 0.46 & -0.992 & 0.035 \\
\cline { 2 - 6 } & RSPH1 & -0.92 & 0.38 & -0.994 & 0.035 \\
\hline rno-miR-17-5p & RPL10L & 0.81 & -1.29 & -0.990 & 0.040 \\
\hline \multirow{2}{*}{$\begin{array}{l}\text { rno-miR-20a +rno- } \\
\text { miR-20b-5p }\end{array}$} & ENG & -0.61 & 0.53 & -0.989 & 0.042 \\
\cline { 2 - 6 } & NOVA2 & -0.61 & 0.36 & -0.989 & 0.043 \\
\hline \multirow{4}{*}{ rno-miR-300-3p } & HYOU1 & -0.55 & 0.45 & -1.000 & 0.035 \\
\cline { 2 - 6 } & MGLL & -0.55 & 0.36 & -0.990 & 0.036 \\
\cline { 2 - 6 } & VASP & -0.55 & 0.33 & -0.992 & 0.035 \\
\hline \multirow{3}{*}{ rno-miR-410 } & FANCG & -0.58 & 0.66 & -0.990 & 0.038 \\
\cline { 2 - 6 } & FAM98A & -0.58 & 0.25 & -0.994 & 0.035 \\
\cline { 2 - 6 } & ENAH & -0.58 & 0.21 & -0.991 & 0.035 \\
\hline rno-miR-451 & XBP1 & -0.50 & 0.46 & -0.993 & 0.035 \\
\cline { 2 - 6 } & RSPH1 & -0.50 & 0.38 & -0.992 & 0.035 \\
\hline rno-miR-499 & EPHB4 & -0.46 & 0.31 & -0.988 & 0.049 \\
\hline
\end{tabular}

Table 1. Significant correlations between miRNAs in fetal plasma and transcripts in fetal brain. miRNAs that were significantly altered in fetal plasma following gestational hypoxia were probed for negative correlation with mRNA that showed significantly changed levels in the fetal brain following the hypoxic insult. $p$ values were adjusted for multiple comparisons using the Benjamini-Hochberg method.

lack of effect of drug treatment on oxidative stress in the fetal liver or brain compared to maternal liver and the placenta. As large NPs $(180 \mathrm{~nm})$ were used, these observations correlate with previous findings that the passage of intravenously injected NPs across the placenta depends on their size ${ }^{62}$. MitoQ-NP treatment was also found to reduce oxidative stress in the placenta of control animals, which had not been exposed to hypoxia, as well as in maternal tissues. The lack of gross changes in the dams and control fetuses in response to maternal MitoQ-NP injection suggests that the treatment did not have adverse effects. However, a more detailed assessment is required to comprehensively investigate if MitoQ-NP administration could adversely, or beneficially, affect maternal health or fetal health in pregnancies without placental oxidative stress.

Furthermore, we observed that maternal MitoQ-NP treatment prevented some abnormal changes in neurons in response to gestational hypoxia. Shortening of dendrites and a reduction in the density of immunostaining of NMDA receptor subunit GluN1 were detected in response to hypoxia. Similar types of change have been reported in neuropsychiatric disease such as schizophrenia or autism ${ }^{13}, 15,17-22$. While shortening or lengthening of TH-positive processes has not been reported in neuropsychiatric disease, both schizophrenia and autism have been associated with increased tyrosine hydroxylase staining in substantia nigra ${ }^{17}$ as well as abnormal dopamine and dopaminergic receptor levels in substantia nigra, striatum and prefrontal cortex ${ }^{17,18}$. The offspring brain in the presented study also showed a loss of parvalbumin-containing cells in cortex and reticular nucleus, which has been linked to schizophrenia and ASD-like behaviours ${ }^{19,20}$, and a reduction in astrocyte/neuron ratio. Previous studies of neurodevelopmental disease have suggested that astrocyte number may be differently affected in different regions of the brain ${ }^{23}$. As it is known that males show higher vulnerability to several neurodevelopmental disorders, including schizophrenia and autism ${ }^{63}$, it would be of future interest to investigate differences in anatomical changes between male and female offspring brains following hypoxia and MitoQ-NP treatment and to establish if the treatment may be more beneficial in one sex over the other.

In addition to the neuroanatomical changes observed in offspring brains, changes in gene expression, among those genes involved in developmental processes, were detected within the fetal brain, in response to gestational hypoxia and MitoQ-NP treatment. Due to the lack of oxidative stress in the fetal brain following gestational hypoxia, the observed neuroanatomical and gene expression changes are less likely caused by a direct effect of the altered environmental oxygen and suggest an indirect effect of maternal hypoxia, potentially via the placenta. The observation that many of the morphological changes in the offspring brain were replicated ex vivo in cortical cultures exposed to culture medium conditioned by rat placenta or to fetal blood support the hypothesis that signalling from the placenta may play a role in neurodevelopment in utero. This is in keeping with previous findings showing that cortical cultures exposed to culture media conditioned by placenta following ex vivo hypoxia/ reoxygenation, had shorter dendrites and altered levels of glutamate receptors ${ }^{43}$. The observation that the effects of fetal blood and conditioned media on neurons could be prevented by maternal treatment with MitoQ-NPs further supports an involvement of the placenta, as MitoQ-NPs were localised and exerted their biological effects on oxidative stress in the placenta and not in the fetus. Moreover, the damaging effects of placental secretions were prevented six days after maternal injection with MitoQ-NPs.

The effects on cortical cultures were also replicated following controlled hypoxic exposure in a simple in vitro barrier model consisting of cytotrophoblast cells, which in the human face the fetal circulation. This suggests that oxidative stress in the placental barrier may mediate effects on the offspring brain, as other factors, such as 

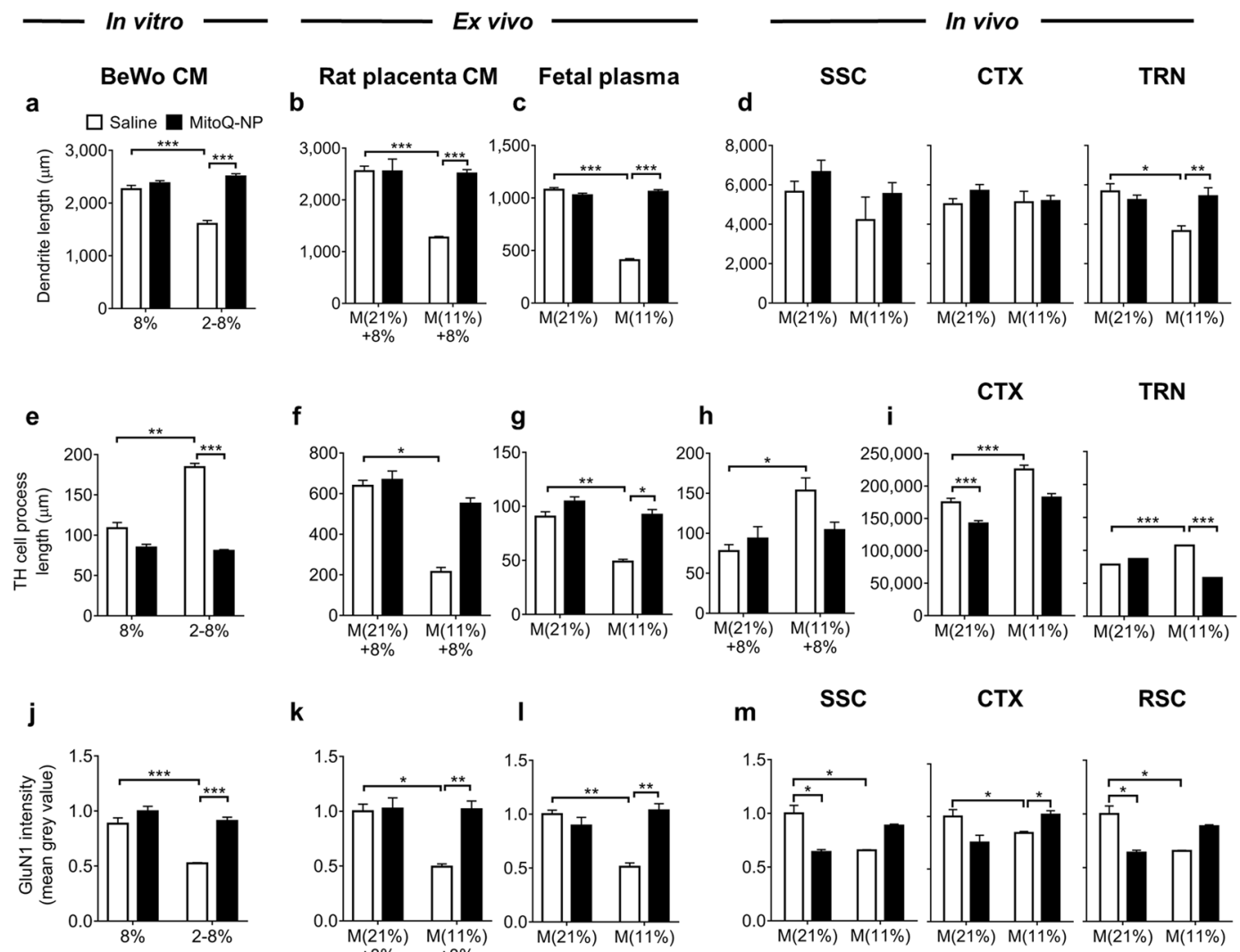

k
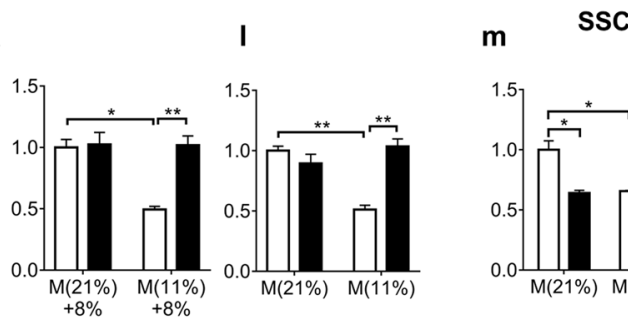

SSC

CTX

RSC

n

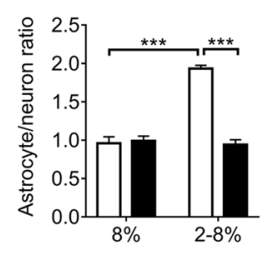

0

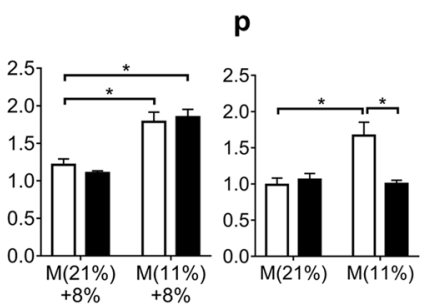

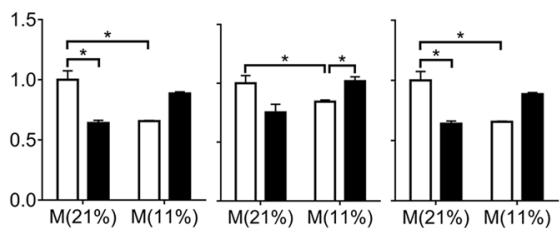

HPC q

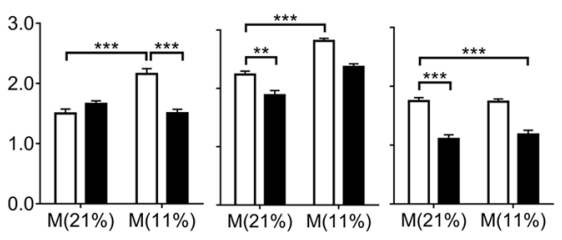

SSC

CTX

TRN

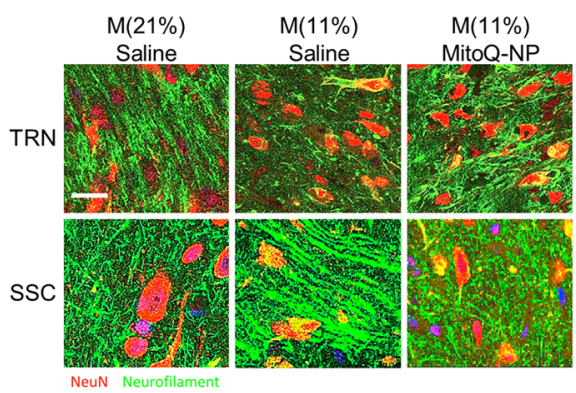

$\mathbf{s}$

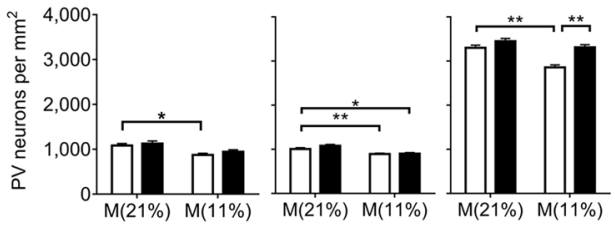

Figure 5. Effects of MitoQ-NPs on neurons. Effects of gestational hypoxia and MitoQ-NP treatment on neurons were examined in vitro by applying culture media conditioned by bilayered BeWo barriers or offspring placenta, or by applying fetal plasma to cortical cultures. Placental tissue and fetal plasma were collected from animals exposed to $6 \mathrm{~d}$ gestational normoxia, $\mathrm{M}(21 \%)$, or gestational hypoxia $\mathrm{M}(11 \%)$, in combination with maternal injection of either saline or MitoQ-NPs. Placental tissue was subsequently incubated ex vivo for $24 \mathrm{~h}$ under $8 \%$ oxygen conditions before collection of conditioned medium. BeWo barriers were cultured at $8 \%$ oxygen to mimic physiological oxygen conditions or at $2 \%$ oxygen followed by $8 \%$ oxygen to mimic hypoxia-rexoygenation (2-8\%). (a-d) Dendrite lengths in vitro following exposure to BeWo conditioned medium (a; number of biological replicates from left to right: $n=25,25,15,15)$, placenta conditioned medium (b; $n=15,10,8,24)$ or plasma (c; $n=21,18,23,18)$ and in vivo $(\mathbf{d})$ in somatosensory cortex (SSC; $n=8$ different brains), combined somatosensory, auditory and retrosplenial cortex (CTX; $n=21,23,21,23$ ) or thalamic reticular nucleus (TRN; $n=8)$. (e-i) Process lengths of tyrosine hydroxylase (TH)-positive neurons in vitro following exposure to BeWo conditioned medium (e; $n=25,25,15,15)$, placenta conditioned medium $(\mathbf{f} ; n=12,10,8,10)$, plasma (g; $n=21,27,19,19)$ or in neuron only-cultures following exposure to placenta 
conditioned medium (h; $n=5,5,5,5)$. (i), $\mathrm{TH}^{+}$process length in vivo in CTX $(n=121,108,123,98)$ and TRN $(n=63,56,59,43)$. (j-m) GluN1 receptor subunit staining intensity in vitro following exposure to BeWo conditioned medium $(\mathbf{j} ; n=15,15,8,9)$, placenta conditioned medium $(\mathbf{k} ; n=10,10,15,10)$ or plasma $(\mathbf{l}$; $n=28,10,17,18)$, and in vivo $(\mathrm{m})$ in SSC $(n=3)$, CTX $(n=3)$ and retrosplenial cortex (RSC; $n=3)$. (n-q) Ratio of astrocytes to neurons in vitro following exposure to BeWo conditioned medium $(\mathbf{n} ; n=15,15,18,12)$, placenta conditioned medium $(\mathbf{o} ; n=10)$ or plasma $(\mathbf{p} ; n=5)$ and in vivo $(\mathbf{q})$ in hippocampus (HPC, $n=23,18,18,24)$, CTX $(n=70,66,72,77)$ and TRN $(n=32,40,44,28)$. (r) Representative images of neurons in TRN and SSC. Sections were stained for neuronal marker NeuN (red) and somato-dendritic marker neurofilament (green). Scale bar $=25 \mu \mathrm{m}$. (s) Density of parvalbumin (PV)-positive neurons in vivo in SSC $(n=21,42,42,33)$, CTX $(n=63,126,126,98)$ and TRN $(n=35,51,55,43) . * p<0.05, * * p<0.01, * * * p<0.001$, Tukey's test following ANOVA.

hormones that could be altered by the maternal hypoxia would not have been present in the in vitro model. We also observed a potential role of astrocytes in mediating the effects of gestational hypoxia and MitoQ-NP treatment. It would therefore be of interest to test whether molecules in fetal blood which were altered by gestational hypoxia could have actions in the brain across a fetal blood-brain barrier via astrocytic end feet.

Analysis of factors in fetal blood and secreted from the placenta ex vivo, showed that the miRNA profile was significantly altered by gestational hypoxia and partially 'normalised' by maternal injection of MitoQ-NPs. miRNAs, small RNAs that function as post-transcriptional regulators, have been shown to play an important role in neurodevelopment ${ }^{56,64}$, brain function ${ }^{65}$ and psychiatric diseases ${ }^{50,56,66}$. Various circulating miRNAs are being studied in patient blood samples in order to develop biomarkers for psychiatric disease ${ }^{66}$. Schizophrenia has generally been associated with an increase in the miRNA biogenesis machinery and with a broad upregulation of cortical miRNAs ${ }^{67}$. Intriguingly, predicted targets of miRNAs significantly altered by gestational hypoxia in fetal blood and conditioned media were found to be enriched within CNVs associated with schizophrenia. Furthermore, a selection of miRNAs were negatively correlated with altered gene expression in the fetal brain. As an example, miR-17-5p was significantly upregulated following gestational hypoxia in both fetal blood and media conditioned by rat placenta; it was also significantly downregulated in response to maternal MitoQ-NP treatment. miR-17-5p upregulation was associated with the downregulation of ribosomal protein L10-like (RPL10L), a ribosomal-like protein with unknown function, in the fetal brain. The miR-17 family of miRNAs plays a role in early neurodevelopment by regulating cell proliferation and differentiation in the cortex ${ }^{68}$. Moreover, an increase in miR-17-5p levels in the human prefrontal cortex has been associated with schizophrenia ${ }^{69,70}$. The results indicate that miRNAs secreted by the placenta could play a role in mediating the effects of gestational hypoxia and drug treatment on the developing fetus and suggest a tentative link between secreted miRNAs and gene expression in the fetal brain. While RNA-loaded extracellular vesicles have been shown to be able to cross the blood-brain barrier into the brain ${ }^{57}$ and extracellular miRNAs have been shown to be functionally active after uptake in target cells in the brain $^{71}$, the hypothesis that placenta-secreted miRNAs may regulate gene expression in the fetal brain remains to be tested.

The proteomic profiles in fetal blood and conditioned media were also altered in response to gestational hypoxia and MitoQ-NP treatment, respectively. Especially lipoprotein-related proteins were found to be differentially abundant in foetal blood, which is of interest in view of the susceptibility of offspring to cardiovascular disease following gestational hypoxia ${ }^{45,72}$. While an interplay between several different factors may be involved, the data strengthen the hypothesis of a mechanistic link between molecules secreted from the placenta, including miRNAs, and the effects of gestational hypoxia on the offspring brain. In some cases differential effects of hypoxia and drug treatment on the secretions in the fetal blood and in media conditioned by rat placenta were observed. A difference between the protein/miRNA profile of fetal blood and conditioned media is to be expected and this difference may reflect the complex interplay between molecules transported across the placenta, molecules secreted by the other fetal tissues, such as fetal liver, into fetal blood and molecules in the fetal blood that are regulated by the placenta. None of these factors would be present in the ex vivo preparation. In contrast, the protein/miRNA profile of culture medium conditioned by rat placenta would consist only of molecules released by the placenta, but in both directions, i.e. to the fetal and the maternal side.

The placenta has been shown to play a key role in fetal programming ${ }^{21,27-32}$. The presented data suggest that treating the placenta with an antioxidant may prevent some changes in our models in response to hypoxia that are also considered to be important in the aetiology of human psychological disorders. Clinical trials with antioxidants in human disease have generally been disappointing, perhaps because not enough antioxidant reaches the mitochondria ${ }^{73-77}$. MitoQ has the advantage of being recycled back to its active form after detoxification of a free radical $^{78,79}$. Moreover, in our rat model MitoQ-NPs were able to produce long-lasting effects following administration of a single controlled dose whilst only affecting the placenta and not the fetus. We propose a future possibility of treating the placenta, and not the fetus, in order to reduce the potential for disease in later life.

\section{Methods}

All animal procedures were conducted in accordance with the United Kingdom Animals Scientific Procedures Act (1986) following institutional ethical approval at the University of Birmingham and then carried out under Home Office project licence authority. Procedures conducted in Canada were approved by the University of Alberta Health Sciences Animal Policy and Welfare Committee, in accordance with the Canadian Council on Animal Care guidelines. All efforts were made to minimize any suffering and the number of animals used. All methods were performed in accordance with the relevant guidelines and regulation. 


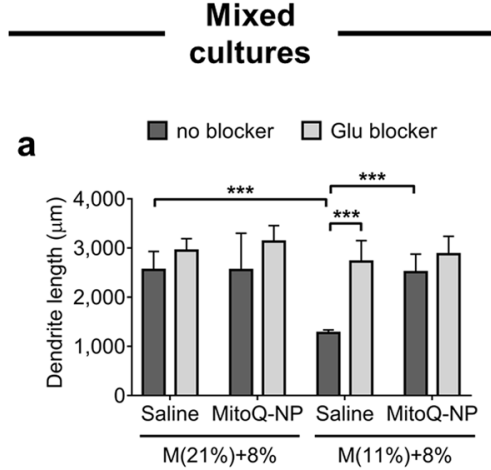

d

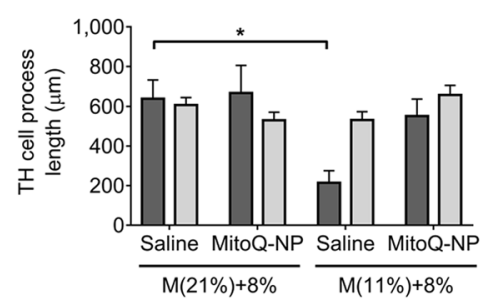

g

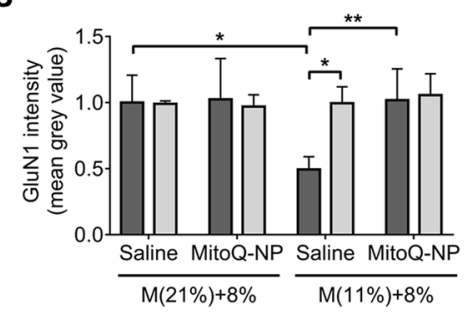

Neuron-only cultures

b

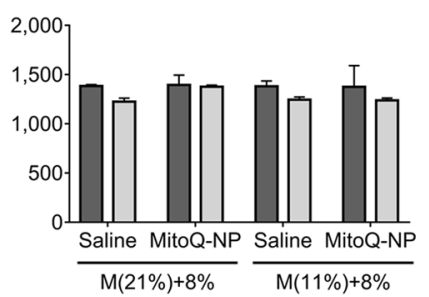

e

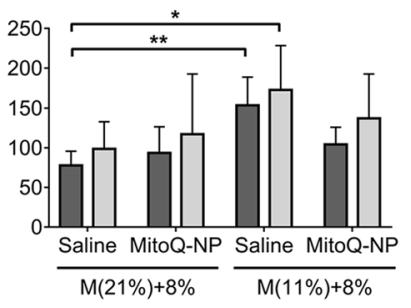

h

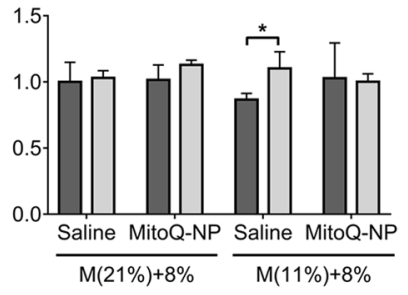

Astrocyte medium on neurons

C

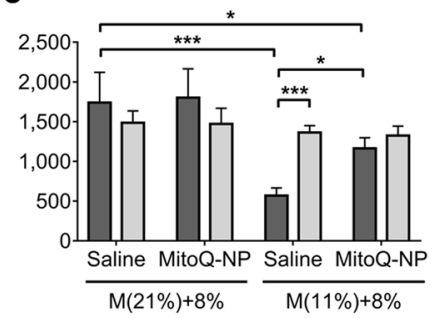

f

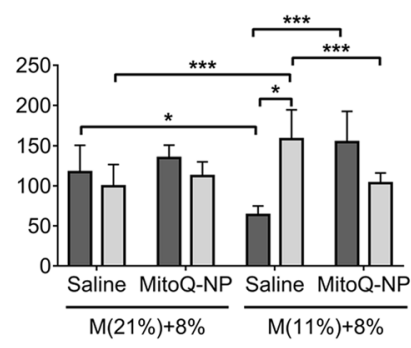

i

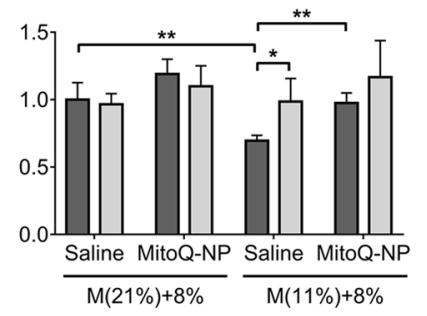

Figure 6. Roles of astrocytes and glutamate signalling in mediating the effects of maternal hypoxia and MitoQ-NPs on neurodevelopment. Mixed cortical cultures (a,d,g) or neuron-only cortical cultures (b,e,h) were incubated with rat placenta conditioned media. The latter were also incubated with medium conditioned by astrocytes which had previously been exposed to rat placenta conditioned media (c,f,i). Placentas were collected from pregnant rats that had been exposed to normoxia, $M(21 \%)$, or hypoxia, $M(11 \%)$, in combination with an injection of saline or MitoQ-NP. This was followed by incubation of the placenta in culture medium for $24 \mathrm{~h}$ ex vivo at $8 \%$ oxygen. To some of the cortical cultures NMDA receptor antagonist MK- 801 was added with the conditioned medium. (a-c) Dendrite lengths of neurons (number of biological replicates from left to right: a, $n=5 ; \mathbf{b}, n=5,4,5,3,3,3 ; \mathbf{c}, n=5 ; \mathbf{d}, n=10)$. (d-f) Dendrite lengths of tyrosine hydroxylase (TH)-positive neurons $(n=5,3,5,3,3,3)$. (g-i) Staining intensity of GluN1 receptor subunits $(\mathbf{e}, n=7,9,5,7 ; \mathbf{f}, n=3 ; \mathbf{g}$, $n=5 ; \mathbf{h}, n=12,10,10,11,25,10)$. Significant differences determined using Tukey's test following ANOVA $(* p<0.05, * * p<0.01, * * * p<0.001)$.

Nanoparticles. The production of drug-loaded $\gamma$-PGA-Phe $\mathrm{NP}^{80}$ is described in Supplemental Methods. Both in vitro and in vivo experiments used NPs carrying a final dose of $0.5 \mu \mathrm{M}$ MitoQ.

Cell cultures. Bilayered cell barriers were prepared using BeWo cells, a choriocarcinoma cell line (gift from Dr Margaret Saunders, University of Bristol, UK) ${ }^{40,41}$ grown on Transwell $0.4 \mu \mathrm{m}$ pore polyester inserts (Corning, UK). Culture media for BeWo barriers consisted of Dubecco's Modified Eagle's Medium (DMEM) with F-12 (Sigma-Aldrich) containing 10\% FBS (Thermo Fisher Scientific) and $2 \mathrm{mM}$ L-glutamine (Sigma-Aldrich). Cell lines were routinely tested for mycoplasma contamination. Integrity of cell barriers was tested by applying fluorescein isothiocyanate-conjugated BSA (FITC-BSA; Sigma-Aldrich) at a final concentration of $100 \mu \mathrm{g} / \mathrm{mL}$ to the top of cell barriers in Transwell inserts and an equal concentration of non-conjugated BSA to the medium below barriers. Fluorescence intensity was measured $1 \mathrm{~h}$ later in the media below the barriers and in the insert.

Cortical cultures were prepared from dissociated rat E18 cortical tissue and grown on glass coverslips as described previously ${ }^{43}$, in Gibco Neurobasal media with 1x Gibco B-27 Supplement, 1x antibiotic-antimycotic (all Thermo Fisher Scientific) and $2 \mathrm{mM} \mathrm{L-glutamine.} \mathrm{To} \mathrm{produce} \mathrm{neuron-only} \mathrm{cultures,} 40 \mu \mathrm{M}$ 5 -fluoro-2-deoxyuridine was added to each well at 5 days in vitro. Astrocyte-only cultures were prepared by growing cortical cultures in DMEM with $10 \% \mathrm{FBS}, 2 \mathrm{mM}$ L-glutamine and $1 \mathrm{x}$ antibiotic-antimycotic. Cultures were accepted as astrocyte-only if neuron number was zero in three representative coverslips. The effect of glutamate 
was investigated by adding MK-801, a NMDA receptor antagonist, at a concentration of $10 \mu \mathrm{M}$ to each well and incubating for $4 \mathrm{~h}$ before exposure of the cells to conditioned media. Exposures of cortical cultures were performed at a minimum in triplicate for condition medium from each of 3 placenta (one from each dam), creating a minimum of 9 data points for each condition. At least three different sets of cortical cultures were tested.

Conditioned media. Conditioned tissue culture media were prepared using bilayered barriers described above or using rat placentas isolated from the in vivo experiment (see below). Barriers were grown and tissues were incubated at $21 \%, 8 \%$ or $2 \%$ oxygen in a SCI-tive hypoxia chamber (Baker Ruskinn, USA). This was followed by a $24 \mathrm{~h}$ exposure period, at the start of which the media under the barriers or around the tissue was replaced. Some wells were additionally exposed to NPs above the barrier. The media was then conditioned by the explant or barrier for $24 \mathrm{~h}$ at the same or altered oxygenation. Conditioned media were applied to embryonic cortex for 6 days from day 12 in vitro to test their effects on cell number, dendrite length, tyrosine hydroxylase positive cell processes and glutamate receptors as previously described ${ }^{40,41}$. Cortical cultures were also exposed to conditioned medium collected from astrocyte-only cultures that had been exposed for $24 \mathrm{~h}$ to rat placenta-conditioned medium. Potential leakage of MitoQ from barriers or placenta was measured in the conditioned medium using liquid chromatography-tandem mass spectrometry (LC-MS/MS) ${ }^{81}$.

Immunocytochemistry. Cortical cultures were fixed in supercold methanol $\left(-20^{\circ} \mathrm{C}\right)$, washed with PBS and blocked with 5\% BSA, 5\% NGS in PBS for $30 \mathrm{~min}$. They were incubated with primary antibodies against MAP2 (1:2000, \#188004; Synaptic Systems, Germany), GFAP (1:1000, \#3670; Cell Signalling Technology), tyrosine hydroxylase (1:500, ab112; abcam) and GluN1 (1:500, ab9864; Merck Millipore), overnight at $4^{\circ} \mathrm{C}$. Sections were stained with secondary antibodies Alexa Fluor 488 anti-rabbit IgG, Alexa Fluor 488 anti-mouse IgG or Alexa Fluor 488 anti-guinea pig IgG (all Thermo Fisher Scientific, diluted 1:500) for $2 \mathrm{~h}$ at room temperature under minimal light conditions, washed with PBS and mounted in DAPI mounting media. Five images per coverslip were taken on a confocal microscope (SP2-AOBS, Leica). Analysis of the slides was performed with the experimenter blind to the experimental group. Dendrite lengths were measured using ImageJ. For receptors images were taken at x64 (with oil) on a fluorescence microscope (Leica SP5II) after excitation at $488 \mathrm{~nm}$. Using ImageJ, images were converted to RGB files then measurements were taken of the mean grey value of each image providing an average of the relative intensity of the staining. Measurements were verified by cell counts based on DAPI staining. Background levels of fluorescence were ascertained in the absence of cells, primary antibody and/or secondary antibody.

In vivo experiments. Three-month-old female Sprague-Dawley rats (Charles River, Wilmington, MA) were maintained on ad libitum standard rat chow and tap water in a 12:12-h light-dark cycle and acclimatized before breeding. Day 0 of pregnancy was determined by sperm in a vaginal smear. On gestational day (GD) 15 of pregnancy the rats were injected intravenously with saline (vehicle control) or $125 \mu \mathrm{M}$ MitoQ-NPs and exposed for the next 6 days to $21 \%$ or $11 \%$ oxygen in an A-Chamber (BioSpherix, USA) ${ }^{44}$. Some rats were sacrificed at GD20. EDTA plasma collected from fetuses by decapitation was pooled per litter and flash frozen; placenta, maternal and fetal tissues were collected fresh, flash-frozen or fixed in formal saline. Other rats were allowed to give birth in normal oxygen conditions. Birth-weights were measured and offspring brains examined at postnatal day (P) 30 .

Statistical tests were not used to predetermine sample size. Experimental groups were allocated according to a preassigned schedule, depending on the order in which successful pregnancy was established. From each litter two males and two females were randomly selected for examination at P30. For subsequent immunohistochemistry analysis of rat tissue, the experimenter was blinded to group allocation.

Localisation of NPs. Pregnant rats were injected with $125 \mu \mathrm{M}$ NPs conjugated with Alexa Fluor 488 and culled at GD16 to collect flash-frozen fetal, maternal and placental tissue. Sagittal sections of placental labyrinth and junctional zone, sections of fetal and maternal cortex and liver were produced using a cryostat and viewed with confocal microscopy for the localisation of fluorescent NPs.

Analysis of placental, fetal and maternal tissues. Fixed placentas were processed for paraffin wax histology. Sections were cut at $3 \mu \mathrm{m}$ and stained with haematoxylin and eosin and at $2 \mu \mathrm{m}$ for avidin-biotin immunocytochemistry for CD34 (ab81289; abcam, UK) using a full automated Bond3 immunostaining machine with bond polymer refined detection (Leica) to ensure the same immunostaining method was applied to all sections. Images were captured on a fluorescent microscope (Leica SP5II) and analysed with ImageJ software. Numbers of biological replicates are given in figure legends. For measurements pertaining to placenta vasculature, values for each biological replicate are averages of technical replicates taken from multiple fields of view.

Levels of reactive oxygen species (ROS) were measured in fetal, maternal and placental tissues using the $2^{\prime}, 7^{\prime}$-dichlorofluorescein diacetate (DCFDA) assay. Sagittal sections $(10 \mu \mathrm{m})$ were cut on the cryostat and exposed to $20 \mu \mathrm{M}$ DCFDA solution in HBSS at $37^{\circ} \mathrm{C}$ in a humidifying chamber. After counter-staining with DAPI, slides were immediately imaged using a confocal microscope (excitation and emission wavelengths of $495 \mathrm{~nm}$ and $529 \mathrm{~nm}$, respectively). Using Image-Pro Premier 9.2 (Media Cybernetics, USA), fluorescence levels of DCF, the product of DCFDA deacetylation by cellular esterases and oxidisation by ROS, were quantified in labyrinth and junctional zone of the placenta, maternal and fetal liver, cortex and cerebellum.

Analysis of conditioned media and plasma. BMP4, 6 and 9 were measured in conditioned media using a cell based assay ${ }^{82}$. Amino acids were measured as previously described ${ }^{43}$.

Total RNA was extracted from $200 \mu \mathrm{l}$ conditioned media or $100 \mu \mathrm{l}$ fetal plasma using the miRNeasy Mini Kit and the miRNeasy Serum/Plasma Kit (Qiagen, Germany). Small RNA and miRNA levels were measured using the Small RNA Kit on the 2100 Bioanalyzer (Agilent Technologies) at the University of Bristol Genomics Facility. Levels of individual miRNAs were analysed using the nCounter Rat v1 miRNA Expression Assay or the nCounter Human 
v2 miRNA Expression Assay (NanoString Technologies, USA), which detects 423 or 800 different species-specific miRNAs, respectively. Briefly, $3 \mu \mathrm{l}$ of each undiluted sample were hybridised with barcoded probes and immobilised on an nCounter Cartridge. Barcode signals were counted using the nCounter Digital Analyzer.

Proteomic analysis was performed by the University of Bristol Proteomics Facility (see Supplementary Methods for details). Briefly, samples were depleted of rat albumin, digested with trypsin and labelled with Tandem Mass Tag (TMT) 10Plex reagents (Thermo Fisher Scientific). The labelled samples were fractionated by high $\mathrm{pH}$ reversed-phase chromatography followed by nano LC-MS/MS. The raw data files were processed and quantified using Proteome Discoverer software v1.4 (Thermo Fisher Scientific) and searched against the UniProt Rat database using the SEQUEST algorithm. All peptide data was filtered to satisfy a false discovery rate of $5 \%$.

RNA sequencing. RNA was extracted from $50 \mathrm{mg}$ of fetal frontal cortex tissue at GD20 using the RNeasy Mini kit (Qiagen). RNA quality and integrity was measured on the 2100 Bioanalyzer. mRNA sequencing was performed by Edinburgh Genomics. Libraries were prepared from total RNA samples using the Illumina TruSeq stranded mRNA Sample Preparation Kit. Briefly the polyA RNA from 1 ug of total RNA was captured onto Oligo $\mathrm{d}(\mathrm{T})$ beads, before fragmentation and elution of the polyA RNA. The RNA was reverse transcribed using random primers and the resulting cDNA was double stranded. The cDNAs were ligated with adapters containing unique barcodes for each sample. Libraries were then assessed for size by electrophoresis and quantified by qPCR. The libraries were sequenced by 75 bases paired-end sequencing across two lanes of an Illumina HiSeq 4000. This level of sequencing produced greater than 34 million paired reads per sample. The FASTQ files were generated using the standard Illumina pipeline for bcl2fastq.

Bioinformatic analyses. Differential expression analysis of NanoString miRNA data is detailed in Supplementary Methods. TargetScanHuman v7.0 ${ }^{83}$ was used to create a list of potential (human-equivalent) targets of the significant miRNAs (Total Context Score $<-0.2$ ). Predicted target genes were analysed for pathway enrichment of CNVs associated with schizophrenia, as described by Pocklington et al. ${ }^{58}$. The analysis was applied to the combined ISC + MGS + CLOZUK dataset, comprising a total of 11,355 cases and 16,416 controls. The analyses are based on large, rare CNVs $(>100 \mathrm{~kb}$, frequency $<1 \%)$, as these are both the most robustly called and most enriched in people with schizophrenia. The primary analysis was performed on all CNVs, with secondary analyses performed for deletions and duplications separately. A further analysis was performed conditioning on the 'minimal' gene sets previously shown to capture CNS-related gene set enrichment in this CNV dataset ${ }^{58}$.

Peptide spectra from the proteomics data set were analysed using moderated $t$-statistics from the empirical Bayes method to calculate differential protein abundance. Modified scripts, published by Kammers et al. ${ }^{84}$ and based on the limma package ${ }^{85}$, were used.

We used Tophat ${ }^{86}$ to align RNA sequencing reads to the rat reference genome Rnor_6.0 (GenBank Assembly ID GCA_000001895.4), and HTSeq ${ }^{87}$ to generate read counts from the resulting BAM files. Out of 672 million paired-end reads, nearly 557 million $(82.8 \%)$ concordant pairs mapped to unique locations in the Rn6 version of the genome. Genes were analysed for differential expression and differentially expressed genes were further analysed for interactions with the miRNA data sets (see Supplementary Methods).

Differentially abundant proteins, differentially expressed genes and predicted targets of differentially secreted miRNAs were analysed for enrichment of biological processes, cellular compartments and tissues using the GO-slim feature in PANTHER $11.0^{88,89}$ and the GO Direct and UP_TISSUE features in DAVID 6.8 ${ }^{90,91}$. Venn diagrams were produced using Venny 2.1 (http://bioinfogp.cnb.csic.es/tools/venny/index.html).

Data availability. Raw and processed data files generated from NanoString and RNA sequencing analyses have been deposited in NCBI's Gene Expression Omnibus ${ }^{92}$ and are accessible through GEO Series accession number GSE86410. The MS proteomics data have been deposited to the ProteomeXchange Consortium via the PRIDE partner repository ${ }^{93}$ with the dataset identifiers PXD004955 and PXD004956 for fetal plasma and conditioned medium, respectively.

Immunohistochemistry of P30 brains. At P30 rats were anaesthetised with $4 \%$ isoflurane and perfused with $4 \%$ PFA. Brains were post-fixed in $4 \%$ PFA, placed in $30 \%$ sucrose until sunk and embedded in OCT. Three brains per condition were randomly selected, one from each litter. $12-\mu \mathrm{m}$ cryostat sections were produced and mounted as contiguous triplicates. Brain sections were exposed to cold methanol at $-20^{\circ} \mathrm{C}$ for $10 \mathrm{~min}$ for fixation. For GluN1 staining, sections were fixed in 2\% PFA, followed by permeabilisation in $0.3 \%$ Triton X-100 in PBS for $15 \mathrm{~min}$. All slides were washed in PBS after every incubation step. Slides were blocked with 5\% goat serum (Sigma-Aldrich), $0.3 \%$ Triton X-100 in PBS for $2 \mathrm{~h}$ at $4{ }^{\circ} \mathrm{C}$, followed by an overnight incubation (or $48 \mathrm{~h}$ incubation for GluN1 staining) at $4^{\circ} \mathrm{C}$ in primary antibody in PBS with $1 \%$ BSA, $0.3 \%$ Triton X-100. Antibodies used were raised against MAP2 (1:500, ab32454; abcam), NeuN (1:1000, ab177487; abcam), neurofilament (1:500, ab24575; abcam), GFAP (1:500, \#3670; Cell Signaling Technology), parvalbumin (1:500, ab11427; abcam), tyrosine hydroxylase (1:500, ab112; abcam) and GluN1 (1:200, MAB1570, Merck Millipore). Slides were incubated with secondary antibody Alexa Fluor 555 anti-rabbit IgG, Alexa Fluor 488 anti-mouse IgG or Alexa Fluor 568 anti-mouse IgG (Thermo Fisher Scientific) at 1:500 for $2 \mathrm{~h}$ at $4^{\circ} \mathrm{C}$. Vectashield Mounting Medium with DAPI (Vector Laboratories, USA) was used to mount coverslips. Coronal sections were chosen to show thalamic reticular nucleus, primary somatosensory, primary auditory and retrosplenial granular cortex ${ }^{94}$. Damaged sections, due to problems with freezing or storage of the samples, were excluded from analysis after visual confirmation. Analysis of brain sections was done with the experimenter blind to the in vivo exposure. For each site, in each brain, a minimum of 5 fields of view were examined for each of 3 sections in both hemispheres using a LASX (Leica) widefield microscope or a SP5II (Leica) confocal microscope at $x 40$ magnification using oil. This resulted in a minimum of 30 fields of view being analysed for each site. The analysis of the intensity of the IHC staining 
of GluN1 was determined using a similar approach to that used previously ${ }^{95}$. Briefly, nine sections per condition were imaged and 10-12 images were collected for every brain region per case and using the same microscopy settings of intensity and magnification to allow for a meaningful comparison. Photos were sorted and converted to greyscale then the total pixel number was determined using a corrected macro for ImageJ. The total pixel number was subsequently subtracted from the background.

Statistics. Data are presented as means \pm s.e.m. For all statistical comparisons, variances were similar in magnitude between the compared groups. As indicated in the figure legends, one-way or two-way ANOVA was performed in Prism 6.0 (GraphPad, USA) or SPSS 21.0 (IBM Corp., USA) with post-hoc analysis using Bonferroni correction or Tukey's test for multiple comparisons. Two-way ANOVA was used to test for main effects of drug and of oxygen conditions and for interaction effects. For GluN1 staining intensity analysis, normality of data distribution was tested using the D'Agostino-Omnibus normality test in Prism $6.0(p>0.05)$ and parametric testing was applied to test for differences of means between groups using multiple unpaired, two-sided Student's $t$-test post-hoc testing adjusted for multiple comparisons using Bonferroni correction.

\section{References}

1. Barker, D. J. The fetal and infant origins of adult disease. BMJ 301, 1111 (1990).

2. Faa, G. et al. Fetal programming of neuropsychiatric disorders. Birth Defects Res C Embryo Today 108, 207-223, doi:10.1002/ bdrc.21139 (2016).

3. Heyer, D. B. \& Meredith, R. M. Environmental toxicology: Sensitive periods of development and neurodevelopmental disorders. Neurotoxicology 58, 23-41, doi:10.1016/j.neuro.2016.10.017 (2016).

4. Fatemi, S. H. \& Folsom, T. D. The neurodevelopmental hypothesis of schizophrenia, revisited. Schizophr Bull 35, 528-548, doi:10.1093/schbul/sbn187 (2009).

5. Nicodemus, K. K. et al. Serious obstetric complications interact with hypoxia-regulated/vascular-expression genes to influence schizophrenia risk. Mol Psychiatry 13, 873-877, doi:10.1038/sj.mp.4002153 (2008).

6. Schmitt, A., Malchow, B., Hasan, A. \& Falkai, P. The impact of environmental factors in severe psychiatric disorders. Front Neurosci 8, 19, doi:10.3389/fnins.2014.00019 (2014).

7. Koga, M., Serritella, A. V., Sawa, A. \& Sedlak, T. W. Implications for reactive oxygen species in schizophrenia pathogenesis. Schizophr Res 176, 52-71, doi:10.1016/j.schres.2015.06.022 (2016).

8. Bayer, T. A., Falkai, P. \& Maier, W. Genetic and non-genetic vulnerability factors in schizophrenia: the basis of the "two hit hypothesis". J Psychiatr Res 33, 543-548 (1999).

9. Coyle, J. T. NMDA receptor and schizophrenia: a brief history. Schizophr Bull 38, 920-926, doi:10.1093/schbul/sbs076 (2012).

10. Garey, L. When cortical development goes wrong: schizophrenia as a neurodevelopmental disease of microcircuits. $J$ Anat $\mathbf{2 1 7}$, 324-333, doi:10.1111/j.1469-7580.2010.01231.x (2010).

11. Belichenko, P. V., Oldfors, A., Hagberg, B. \& Dahlström, A. Rett syndrome: 3-D confocal microscopy of cortical pyramidal dendrites and afferents. Neuroreport 5, 1509-1513 (1994).

12. Armstrong, D., Dunn, J. K., Antalffy, B. \& Trivedi, R. Selective dendritic alterations in the cortex of Rett syndrome. J Neuropathol Exp Neurol 54, 195-201 (1995).

13. Hutsler, J. J. \& Zhang, H. Increased dendritic spine densities on cortical projection neurons in autism spectrum disorders. Brain Res 1309, 83-94, doi:10.1016/j.brainres.2009.09.120 (2010).

14. Tang, G. et al. Loss of mTOR-dependent macroautophagy causes autistic-like synaptic pruning deficits. Neuron 83, 1131-1143, doi:10.1016/j.neuron.2014.07.040 (2014).

15. Garey, L. J. et al. Reduced dendritic spine density on cerebral cortical pyramidal neurons in schizophrenia. J Neurol Neurosurg Psychiatry 65, 446-453 (1998).

16. Chapleau, C. A. et al. Dendritic spine pathologies in hippocampal pyramidal neurons from Rett syndrome brain and after expression of Rett-associated MECP2 mutations. Neurobiol Dis 35, 219-233, doi:10.1016/j.nbd.2009.05.001 (2009).

17. Howes, O., McCutcheon, R. \& Stone, J. Glutamate and dopamine in schizophrenia: an update for the 21st century. J Psychopharmacol 29, 97-115, doi:10.1177/0269881114563634 (2015).

18. Kriete, T. \& Noelle, D. C. Dopamine and the development of executive dysfunction in autism spectrum disorders. PLoS One 10, e0121605, doi:10.1371/journal.pone.0121605 (2015).

19. Rotaru, D. C., Lewis, D. A. \& Gonzalez-Burgos, G. The role of glutamatergic inputs onto parvalbumin-positive interneurons: relevance for schizophrenia. Rev Neurosci 23, 97-109, doi:10.1515/revneuro-2011-0059 (2012).

20. Wöhr, M. et al. Lack of parvalbumin in mice leads to behavioral deficits relevant to all human autism core symptoms and related neural morphofunctional abnormalities. Transl Psychiatry 5, e525, doi:10.1038/tp.2015.19 (2015).

21. Rapoport, J. L., Giedd, J. N. \& Gogtay, N. Neurodevelopmental model of schizophrenia: update 2012. Mol Psychiatry 17, 1228-1238, doi:10.1038/mp.2012.23 (2012)

22. Lee, E. J., Choi, S. Y. \& Kim, E. NMDA receptor dysfunction in autism spectrum disorders. Curr Opin Pharmacol 20, 8-13, doi:10.1016/j.coph.2014.10.007 (2015).

23. Molofsky, A. V. et al. Astrocytes and disease: a neurodevelopmental perspective. Genes Dev 26, 891-907, doi:10.1101/gad.188326.112 (2012).

24. Xia, M., Abazyan, S., Jouroukhin, Y. \& Pletnikov, M. Behavioral sequelae of astrocyte dysfunction: focus on animal models of schizophrenia. Schizophr Res 176, 72-82, doi:10.1016/j.schres.2014.10.044 (2016).

25. Vargas, D. L., Nascimbene, C., Krishnan, C., Zimmerman, A. W. \& Pardo, C. A. Neuroglial activation and neuroinflammation in the brain of patients with autism. Ann Neurol 57, 67-81, doi:10.1002/ana.20315 (2005).

26. Suzuki, K. et al. Microglial activation in young adults with autism spectrum disorder. JAMA Psychiatry 70, 49-58, doi:10.1001/ jamapsychiatry.2013.272 (2013).

27. Edwards, C. R., Benediktsson, R., Lindsay, R. S. \& Seckl, J. R. Dysfunction of placental glucocorticoid barrier: link between fetal environment and adult hypertension? Lancet 341, 355-357 (1993).

28. Godfrey, K. M. The role of the placenta in fetal programming-a review. Placenta 23(Suppl A), S20-27, doi:10.1053/plac.2002.0773 (2002).

29. Jansson, T. \& Powell, T. L. IFPA 2005 Award in Placentology Lecture. Human placental transport in altered fetal growth: does the placenta function as a nutrient sensor?-a review. Placenta 27(Suppl A), S91-97, doi:10.1016/j.placenta.2005.11.010 (2006).

30. Hsiao, E. Y. \& Patterson, P. H. Placental regulation of maternal-fetal interactions and brain development. Dev Neurobiol 72, 1317-1326, doi:10.1002/dneu.22045 (2012).

31. Stolp, H., Neuhaus, A., Sundramoorthi, R. \& Molnár, Z. The Long and the Short of it: Gene and Environment Interactions During Early Cortical Development and Consequences for Long-Term Neurological Disease. Front Psychiatry 3, 50, doi:10.3389/ fpsyt.2012.00050 (2012) 
32. Mikaelsson, M. A., Constância, M., Dent, C. L., Wilkinson, L. S. \& Humby, T. Placental programming of anxiety in adulthood revealed by Igf2-null models. Nat Commun 4, 2311, doi:10.1038/ncomms3311 (2013).

33. Bonnin, A. et al. A transient placental source of serotonin for the fetal forebrain. Nature 472, 347-350, doi:10.1038/nature09972 (2011).

34. McKay, R. Developmental biology: Remarkable role for the placenta. Nature 472, 298-299, doi:10.1038/472298a (2011).

35. Goeden, N. et al. Maternal Inflammation Disrupts Fetal Neurodevelopment via Increased Placental Output of Serotonin to the Fetal Brain. J Neurosci 36, 6041-6049, doi:10.1523/JNEUROSCI.2534-15.2016 (2016).

36. Dalman, C., Allebeck, P., Cullberg, J., Grunewald, C. \& Köster, M. Obstetric complications and the risk of schizophrenia: a longitudinal study of a national birth cohort. Arch Gen Psychiatry 56, 234-240 (1999).

37. Walker, C. K. et al. Preeclampsia, placental insufficiency, and autism spectrum disorder or developmental delay. JAMA Pediatr 169, 154-162, doi:10.1001/jamapediatrics.2014.2645 (2015).

38. Mann, J. R. \& McDermott, S. Are maternal genitourinary infection and pre-eclampsia associated with ADHD in school-aged children? J Atten Disord 15, 667-673, doi:10.1177/1087054710370566 (2011).

39. Getahun, D. et al. In utero exposure to ischemic-hypoxic conditions and attention-deficit/hyperactivity disorder. Pediatrics 131, e53-61, doi:10.1542/peds.2012-1298 (2013).

40. Bhabra, G. et al. Nanoparticles can cause DNA damage across a cellular barrier. Nat Nanotechnol 4, 876-883, doi:10.1038/ nnano.2009.313 (2009).

41. Sood, A. et al. Signalling of DNA damage and cytokines across cell barriers exposed to nanoparticles depends on barrier thickness. Nat Nanotechnol 6, 824-833, doi:10.1038/nnano.2011.188 (2011).

42. Jones, A. J. et al. Evidence for bystander signalling between human trophoblast cells and human embryonic stem cells. Sci Rep 5, 11694, doi:10.1038/srep11694 (2015).

43. Curtis, D. J. et al. Secretions from placenta, after hypoxia/reoxygenation, can damage developing neurones of brain under experimental conditions. Exp Neurol 261, 386-395, doi:10.1016/j.expneurol.2014.05.003 (2014).

44. Morton, J. S., Rueda-Clausen, C. F. \& Davidge, S. T. Flow-mediated vasodilation is impaired in adult rat offspring exposed to prenatal hypoxia. J Appl Physiol (1985) 110, 1073-1082, doi:10.1152/japplphysiol.01174.2010 (2011).

45. Giussani, D. A. \& Davidge, S. T. Developmental programming of cardiovascular disease by prenatal hypoxia. J Dev Orig Health Dis 4, 328-337, doi:10.1017/S204017441300010X (2013)

46. Casas-Agustench, P., Iglesias-Gutiérrez, E. \& Dávalos, A. Mother's nutritional miRNA legacy: Nutrition during pregnancy and its possible implications to develop cardiometabolic disease in later life. Pharmacol Res 100, 322-334, doi:10.1016/j.phrs.2015.08.017 (2015).

47. Brett, K. E., Ferraro, Z. M., Yockell-Lelievre, J., Gruslin, A. \& Adamo, K. B. Maternal-fetal nutrient transport in pregnancy pathologies: the role of the placenta. Int J Mol Sci 15, 16153-16185, doi:10.3390/ijms150916153 (2014).

48. Cetin, I. \& Alvino, G. Intrauterine growth restriction: implications for placental metabolism and transport. A review. Placenta 30(Suppl A), S77-82, doi:10.1016/j.placenta.2008.12.006 (2009).

49. Pignatti, E., Zeller, R. \& Zuniga, A. To BMP or not to BMP during vertebrate limb bud development. Semin Cell Dev Biol 32, 119-127, doi:10.1016/j.semcdb.2014.04.004 (2014).

50. Geaghan, M. \& Cairns, M. J. MicroRNA and Posttranscriptional Dysregulation in Psychiatry. Biol Psychiatry 78, 231-239, doi:10.1016/j.biopsych.2014.12.009 (2015)

51. Kalia, M. \& Costa, E. S. J. Biomarkers of psychiatric diseases: current status and future prospects. Metabolism 64, S11-15, doi:10.1016/j.metabol.2014.10.026 (2015).

52. Pietersen, C. Y. et al. Molecular profiles of pyramidal neurons in the superior temporal cortex in schizophrenia. J Neurogenet 28, 53-69, doi:10.3109/01677063.2014.882918 (2014).

53. Li, J. Y., Yong, T. Y., Michael, M. Z. \& Gleadle, J. M. MicroRNAs: are they the missing link between hypoxia and pre-eclampsia? Hypertens Pregnancy 33, 102-114, doi:10.3109/10641955.2013.832772 (2014).

54. Huang, Y. C. et al. Characterization of MSCs from human placental decidua basalis in hypoxia and serum deprivation. Cell Biol Int 34, 237-243, doi:10.1042/CBI20090044 (2010).

55. Huntzinger, E. \& Izaurralde, E. Gene silencing by microRNAs: contributions of translational repression and mRNA decay. Nat Rev Genet 12, 99-110, doi:10.1038/nrg2936 (2011).

56. Sun, E. \& Shi, Y. MicroRNAs: Small molecules with big roles in neurodevelopment and diseases. Exp Neurol 268, 46-53, doi:10.1016/j.expneurol.2014.08.005 (2015).

57. Alvarez-Erviti, L. et al. Delivery of siRNA to the mouse brain by systemic injection of targeted exosomes. Nat Biotechnol 29, 341-345, doi:10.1038/nbt.1807 (2011)

58. Pocklington, A. J. et al. Novel Findings from CNVs Implicate Inhibitory and Excitatory Signaling Complexes in Schizophrenia. Neuron 86, 1203-1214, doi:10.1016/j.neuron.2015.04.022 (2015).

59. Kapur, S. Psychosis as a state of aberrant salience: a framework linking biology, phenomenology, and pharmacology in schizophrenia. Am J Psychiatry 160, 13-23, doi:10.1176/appi.ajp.160.1.13 (2003).

60. Hasegawa-Ishii, S. et al. Endotoxemia-induced cytokine-mediated responses of hippocampal astrocytes transmitted by cells of the brain-immune interface. Sci Rep 6, 25457, doi:10.1038/srep25457 (2016).

61. Barker, D. J. The developmental origins of adult disease. J Am Coll Nutr 23, 588S-595S (2004).

62. Semmler-Behnke, M. et al. Size dependent translocation and fetal accumulation of gold nanoparticles from maternal blood in the rat. Part Fibre Toxicol 11, 33, doi:10.1186/s12989-014-0033-9 (2014).

63. Bale, T. L. The placenta and neurodevelopment: sex differences in prenatal vulnerability. Dialogues Clin Neurosci 18, 459-464 (2016).

64. Follert, P., Cremer, H. \& Beclin, C. MicroRNAs in brain development and function: a matter of flexibility and stability. Front Mol Neurosci 7, 5 (2014).

65. Fiorenza, A. \& Barco, A. Role of Dicer and the miRNA system in neuronal plasticity and brain function. Neurobiol Learn Mem 135, 3-12 (2016).

66. Issler, O. \& Chen, A. Determinig the role of microRNAs in psychiatric disorders. Nat Rev Neurosci 16, 201-212 (2015).

67. Beveridge, N. J., Gardiner, E., Carroll, A. P., Tooney, P. A. \& Cairns, M. J. Schizophrenia is associated with an increase in cortical microRNA biogenesis. Mol Psychiatry 15, 1176-1189 (2010).

68. Mao, S. et al. miR-17 regulates the proliferation and differentiation of the neural precursor cells during mouse corticogenesis. FEBS J 281, 1144-1158 (2014).

69. Santarelli, D. M., Beveridge, N. J., Tooney, P. A. \& Cairns, M. J. Upregulation of dicer and microRNA expression in the dorsolateral prefrontal cortex Broadmann area 46 in schizophrenia. Biol Psychiatry 69, 180-187 (2015).

70. Smalheiser, N. R. et al. Expression of microRNAs and other small RNAs in prefrontal cortex in schizophrenia, bipolar disorder and depressed subjects. PLoS One 9, e86469, doi:10.1371/journal.pone.0086469 (2014).

71. Morel, L. et al. Neuronal exosomal miRNA-dependent translational regulation of astroglial glutamate transporter GLT1. J Biol Chem 288, 7105-7116 (2013).

72. Hutter, D., Kingdom, J. \& Jaeggi, E. Causes and mechanisms of intrauterine hypoxia and its impact on the fetal cardiovascular system: a review. Int J Pediatr 2010, 401323, doi:10.1155/2010/401323 (2010).

73. Roberts, J. M. et al. Vitamins C and E to prevent complications of pregnancy-associated hypertension. N Engl J Med 362, 1282-1291 (2010). 
74. Villar, J. et al. World Health Organisation multicentre randomised trial of supplementation with vitamins $\mathrm{C}$ and $\mathrm{E}$ among pregnant women at high risk for pre-eclampsia in populations of low nutritional status from developing countries. BJOG 116, 780-788 (2009).

75. Spinnato, J. A. et al. Antioxidant therapy to prevent preeclampsia: a randomized controlled trial. Obstet Gynecol 110, 1311-1318 (2007).

76. Rumbold, A. R. et al. Vitamins C and E and the risks of preeclampsia and perinatal complications. N Engl J Med 354, 1796-1806 (2006).

77. Poston, L. et al. Vitamin C and vitamin E in pregnant women at risk for pre-eclampsia (VIP trial): randomised placebo-controlled trial. Lancet 367, 1145-1154 (2006).

78. Kelso, G. F. et al. Selective targeting of a redox-active ubiquinone to mitochondria within cells: antioxidant and antiapoptotic properties. J Biol Chem 276, 4588-4596, doi:10.1074/jbc.M009093200 (2001).

79. James, A. M., Cochemé, H. M., Smith, R. A. \& Murphy, M. P. Interactions of mitochondria-targeted and untargeted ubiquinones with the mitochondrial respiratory chain and reactive oxygen species. Implications for the use of exogenous ubiquinones as therapies and experimental tools. J Biol Chem 280, 21295-21312, doi:10.1074/jbc.M501527200 (2005).

80. Kim, H., Akagi, T. \& Akashi, M. Preparation of size tunable amphiphilic poly(amino acid) nanoparticles. Macromol Biosci 9 , 842-848, doi:10.1002/mabi.200800367 (2009).

81. Rodriguez-Cuenca, S. et al. Consequences of long-term oral administration of the mitochondria-targeted antioxidant MitoQ to wild-type mice. Free Radic Biol Med 48, 161-172, doi:10.1016/j.freeradbiomed.2009.10.039 (2010).

82. Herrera, B. \& Inman, G. J. A rapid and sensitive bioassay for the simultaneous measurement of multiple bone morphogenetic proteins. Identification and quantification of BMP4, BMP6 and BMP9 in bovine and human serum. BMC Cell Biol 10, 20, doi:10.1186/1471-2121-10-20 (2009).

83. Agarwal, V., Bell, G. W., Nam, J. W. \& Bartel, D. P. Predicting effective microRNA target sites in mammalian mRNAs. Elife 4, doi:10.7554/eLife.05005 (2015).

84. Kammers, K., Cole, R. N., Tiengwe, C. \& Ruczinski, I. Detecting Significant Changes in Protein Abundance. EuPA Open Proteom 7 , 11-19, doi:10.1016/j.euprot.2015.02.002 (2015).

85. Ritchie, M. E. et al. limma powers differential expression analyses for RNA-sequencing and microarray studies. Nucleic Acids Res 43, e47, doi:10.1093/nar/gkv007 (2015).

86. Trapnell, C., Pachter, L. \& Salzberg, S. L. TopHat: discovering splice junctions with RNA-Seq. Bioinformatics 25, 1105-1111, doi:10.1093/bioinformatics/btp120 (2009)

87. Anders, S., Pyl, P. T. \& Huber, W. HTSeq - A Python framework to work with high-throughput sequencing data. Bioinformatics 31, 166-169, doi:10.1093/bioinformatics/btu638 (2015).

88. Mi, H., Muruganujan, A., Casagrande, J. T. \& Thomas, P. D. Large-scale gene function analysis with the PANTHER classification system. Nat Protoc 8, 1551-1566, doi:10.1038/nprot.2013.092 (2013).

89. Mi, H., Poudel, S., Muruganujan, A., Casagrande, J. T. \& Thomas, P. D. PANTHER version 10: expanded protein families and functions, and analysis tools. Nucleic Acids Res 44, D336-342, doi:10.1093/nar/gkv1194 (2016).

90. Huang, dW., Sherman, B. T. \& Lempicki, R. A. Bioinformatics enrichment tools: paths toward the comprehensive functional analysis of large gene lists. Nucleic Acids Res 37, 1-13, doi:10.1093/nar/gkn923 (2009).

91. Huang, dW., Sherman, B. T. \& Lempicki, R. A. Systematic and integrative analysis of large gene lists using DAVID bioinformatics resources. Nat Protoc 4, 44-57, doi:10.1038/nprot.2008.211 (2009).

92. Edgar, R., Domrachev, M. \& Lash, A. E. Gene Expression Omnibus: NCBI gene expression and hybridization array data repository. Nucleic Acids Res 30, 207-210 (2002).

93. Vizcaíno, J. A. et al. The PRoteomics IDEntifications (PRIDE) database and associated tools: status in 2013. Nucleic Acids Res 41, D1063-1069, doi:10.1093/nar/gks1262 (2013).

94. Paxinos, G. \& Watson, C. The rat brain in stereotaxic coordinates. 6 edn, (Academic Press, 2006).

95. Hughes, E. G. et al. Cellular and synaptic mechanisms of anti-NMDA receptor encephalitis. J Neurosci 30, 5866-5875, doi:10.1523/ JNEUROSCI.0167-10.2010 (2010).

\section{Acknowledgements}

The authors acknowledge the very generous and valuable support of the Pervoli Trust. The work conducted in the Davidge laboratory is funded by grants from the Canadian Institutes of Health Research (CIHR); and the Women and Children's Health Research Institute (WCHRI) through the generous contributions of the Stollery Children's Hospital Foundation (SCHF) and the Royal Alexandra Hospital Foundation (RAHF). S. Davidge is a Canada Research Chair in Maternal and Perinatal Cardiovascular Health. Computing equipment was supported by an EPSRC grant (EP/K008250/1)

\section{Author Contributions}

T.P., H.S., D.M., A.B., A.S., J.M., T.A., K.A., C.G., G.I., M.A., C.-L.C., B.S., A.L., G.C., H.K., A.C., C.C., did the experiments. T.P., H.S., D.M., A.S., T.A., M.R., A.P., H.K., P.H., M.M., T.F., M.A., C.C., analysed the data. S.G., Y.C., A.C., S.D., M.A. provided materials. T.P., H.S., C.C. wrote the manuscript.

\section{Additional Information}

Supplementary information accompanies this paper at doi:10.1038/s41598-017-06300-1

Competing Interests: H.S. consults for Placentum Ltd. MPM consults for Antipodean Pharmaceuticals Inc. All other authors declare no competing financial interests.

Publisher's note: Springer Nature remains neutral with regard to jurisdictional claims in published maps and institutional affiliations.

Open Access This article is licensed under a Creative Commons Attribution 4.0 International License, which permits use, sharing, adaptation, distribution and reproduction in any medium or format, as long as you give appropriate credit to the original author(s) and the source, provide a link to the Creative Commons license, and indicate if changes were made. The images or other third party material in this article are included in the article's Creative Commons license, unless indicated otherwise in a credit line to the material. If material is not included in the article's Creative Commons license and your intended use is not permitted by statutory regulation or exceeds the permitted use, you will need to obtain permission directly from the copyright holder. To view a copy of this license, visit http://creativecommons.org/licenses/by/4.0/.

(c) The Author(s) 2017 